\title{
Vergleichende Sprachinselforschung: Sprachwandel in deutschen Sprachinseln in Russland und Brasilien Peter Rosenberg (Frankfurt/Oder)
}

\begin{abstract}
Dealing with convergence in German speech islands in Russia, Brazil and the United states the article discusses the linguistic phenomena related to the notion of convergence from different vantage points including intralinguistic convergence (due to dialect-dialect contact), interlinguistic convergence (due to language-language contact), typological "convergence" (or intralinguistic change), pidginization, and cognitive processes of simplification.
\end{abstract}

Most of the German speech islands are considered to be contracting - if not dying - varieties with respect to the reduction of their grammatical systems. Evidently, for a long time language contact (and sometimes variety contact) have severely increased. Linguistic norms have been weakened in terms of both norm certainty and norm loyalty thus giving way to processes similar to those common to pidgin languages.

External induced changes are highly remarkable in all German speech islands. But the susceptibility for change and the ways of change are structured by systematical and typological constraints which probably turn out to be cognitive processes underlying quite "normal" linguistic change. This change is discussed as a subsequent process of "regularization" (of irregular forms), simplification (of rules) and loss of grammatical distinctions (and their compensation).

The linguistic description of these interrelated processes is based on an integrated approach providing methodology from sociolinguistics, dialectology and research on language change, including the attempt to highlight the cognitive structures which furrow the line for internal simplifications under external pressure. Comparative speech island research seems to be a promising field of application for the description of the intermesh of these processes.

\section{$1 \quad$ Aufgaben der Sprachinselforschung}

Warum wandeln sich Sprachen? Wollten wir diese Frage im Sinne Eugenio Coserius beantworten, wäre dieser Aufsatz hier bereits beendet: Nach Coseriu (1974) wäre dies eine sinnlose Frage, denn - so allgemein aufgeworfen - ist sie absurd: Sprachen müssen sich wandeln, um zu funktionieren. Sie jedoch auf 
einer historischen Ebene aufzuwerfen, hieße, sich Rechenschaft abzulegen über die spezifischen sprachlichen Notwendigkeiten und kommunikativen Umstände, die einen sprachlichen Wandel zu einer gegebenen Zeit, an einem gegebenen Ort und unter gegebenen inneren und äußeren Bedingungen verursachen. ${ }^{1}$

Sprachlichen Wandel in Sprachinseln und generell bei Sprachminderheiten zu untersuchen, bedeutet häufig, sich einer verwirrenden Vielfalt von "inneren" und "äußeren" Sprachwandelfaktoren gegenüberzusehen, die es gedanklich zu ordnen und zu interpretieren gilt.

Während für solche Sprachgemeinschaften schnell und gern angenommen wird, grammatischer Wandel sei dann auf intensiven Sprachkontakt zurückzuführen, wenn sich der Wandel in Richtung der Mehrheitssprache vollziehe, hat sich Wayne O'Neil vor einigen Jahren in einem wichtigen, in Europa jedoch wenig beachteten Beitrag die Aufgabe gestellt, intern und extern bedingten grammatischen Wandel gegeneinander abzugrenzen.

\section{Er unterschied zwischen}

"grammatical change in which there is neutralization of the point(s) of difference between two languages in contact and grammatical change involving simplification of a feature of a language, change which is evidently not the result of one language working on another" (O'Neil 1978: 248).

Neutralisation gehe auf Erfordernisse des Sprachkontakts zurück, der ein Bedürfnis "to ease understanding" erzeuge (O'Neil 1978: 275). Simplifikation entspringe dagegen einem "language-internal move to ease learning" (O'Neil 1978: 282). Und er benennt das ungelöste sprachwissenschaftliche Problem: "We cannot, however, explain this simplification" (O'Neil 1978: 248). ${ }^{2}$

Deutsche Sprachinseln sind heute nahezu überall auf der Welt intensivem Sprachkontakt ausgesetzt. Wie noch zu zeigen sein wird, befinden sich die hier gesprochenen Varietäten zugleich häufig in rapidem Wandel, Simplifikationsprozesse im Sinne eines Abbaus morphologischer Strukturen

\footnotetext{
${ }^{1}$ Sprachwandel ist nach Coseriu (1974: 56) auf drei Ebenen zu untersuchen: auf der $r a-$ tionalen Ebene (warum wandeln sich Sprachen?), auf der allgemeinen Ebene (welche Bedingungen verursachen Sprachwandel?), und auf der historischen Ebene (der Untersuchung eines spezifischen Sprachwandelphänomens): Während auf der rationalen Ebene sprachlicher Wandel die Wesensbedingung der Kommunikation sei, sei der Wandel auf der allgemeinen Ebene Ausdruck der veränderlichen Balance des Sprachsystems, im Besonderen des Wechselverhätlnisses von System und Norm (siehe unten). Als historisches Problem sehen wir uns der Aufgabe gegenüber, dieses Wechselverhältnis in einer spezifischen historischen Situation zu analysieren: Sprachwandel wird gefördert durch regionale und soziale Variabilität von Sprachwissen und Spracheinstellungen in Zeiten kultureller Veränderung oder sozialer Divergenz (cf. Coseriu 1974: 100).

${ }^{2}$ Ich komme auf diese offene Frage in Kapitel 4 zurück.
} 
inbegriffen. Sprachwandel und sprachliche Konvergenz überkreuzen sich, und gelegentlich ist es nicht leicht, intern und extern induzierte sprachliche Entwicklungen voneinander abzugrenzen.

"Sprachinseln" ${ }^{3}$ sind Sprachgemeinschaften auf begrenztem Raum mit Sprachen oder Sprachvarietäten, die sich von der Sprache der Umgebung mehr oder weniger deutlich unterscheiden und mit denen ein Bewusstsein der eigenen Distinktivität verbunden ist, getragen durch ein dichtes kommunikatives Netzwerk (im Sinne von Milroy 1980 und Gumperz 1968), das eher nach innen als nach außen gerichtet ist.

Diese "restringierten" Umstände bieten der Forschung den Vorteil der Überschaubarkeit: Faktoren des Sprachwandels und der sprachlichen Konvergenz sind innerhalb einer Sprachinsel oft leichter zu kontrollieren und in ihrem Zusammenspiel zu analysieren.

Eine vergleichende Betrachtung der sprachlichen Entwicklung in Sprachinseln unter sehr unterschiedlichen Sprachkontaktbedingungen kann darüber hinaus dazu beitragen, das Verhältnis von Wandel und Konvergenz zu erhellen und das Ineinandergreifen interner und externer Sprachwandelphänomene zu interpretieren.

Selbstverständlich sind Sprachinseln kein rein deutsches Phänomen. Sprachinseln existieren überall auf der Welt. Sprachinseln sind in der deutschen Sprachwissenschaft jedoch frühzeitig zu einem eigenständigen Forschungsgegenstand erhoben worden. Während sie anderenorts oft unter Fragestellungen der Sprachkontakt- und Sprachminderheitenforschung subsumiert wurden, galt das Interesse der deutschen Sprachinselforschung vorrangig der Erforschung des Sprachwandels.

Die Sprachinselforschung ${ }^{4}$ war über viele Jahrzehnte ein bedeutendes Forschungsfeld der deutschen Dialektologie. Berücksichtigt man, dass die deutsche Dialektologie - nach der Indogermanistik das zweite große Forschungsgebiet der deutschen Sprachwissenschaft im späten 19. und beginnenden 20. Jahrhundert für eine gewisse Zeit international führend war, verwundert es nicht, dass die deutsche Sprachinselforschung allen wichtigen dialektologischen Ansätzen als

\footnotetext{
3 "Sprachinseln sind räumlich abgrenzbare und intern strukturierte Siedlungsräume einer sprachlichen Minderheit inmitten einer anderssprachigen Mehrheit" (Hutterer 1982: 178).

"Sprachinseln sind punktuell oder areal auftretende, relativ kleine geschlossene Sprachund Siedlungsgemeinschaften in einem anderssprachigen, relativ größeren Gebiet" (Wiesinger 1983: 901).

${ }^{4}$ Zur Geschichte der Sprachinselforschung cf. Rosenberg (2003), Mattheier (1996), Kuhn (1934).
} 
methodologisches "Prüffeld" diente: Die Dialektgeographie ${ }^{5}$ ist hier mit ihrer areallinguistischen Methode ebenso vertreten wie die Junggrammatiker ${ }^{6}$ mit einer beträchtlichen Anzahl von Ortsgrammatiken und auch die spätere "Kulturraumforschung" ${ }^{7}$ mit sprachhistorischen Untersuchungen in deutschen Siedlungsgebieten in Ostmitteleuropa.

Sprachinselstudien waren - soweit sie nicht rein deskriptiv arbeiteten - vor allem durch das Interesse an einer Rekonstruktion des Sprachwandels motiviert: Die meisten Sprachinseln waren kleine Sprachgemeinschaften mit begrenzten kommunikativen Aktivitäten nach außerhalb und dadurch umfassend beschreibbar und gut rekonstruierbar in Raum und Zeit. Diese "konservativen" Gemeinschaften mit ihren zahlreichen Archaismen galten als einzigartige Beobachtungsobjekte für die Rekonstruktion von sprachlichen Merkmalen, die im geschlossenen deutschen Sprachgebiet bereits im Rückzug begriffen oder sogar ausgestorben waren: Die Beschreibung sprachlichen Wandels verlangte nach Vergleichsmustern, die alte Zustände repräsentierten. Der Mythos der "Reinheit" und "Homogenität" der Sprachinselvarietäten begründete dabei vielleicht mehr als alles andere ihre Attraktivität. Sprachliche Variation ebenso wie äußere Einflüsse durch Sprachkontakt wurden folgerichtig eher als Kontamination gesehen, als zum eigenständigen Forschungsgegenstand erhoben zu werden.

Untersuchungen der ersten Forschungsperiode wurden meist von Dialektologen aus dem deutschen Binnensprachraum durchgeführt, selten von Forschern aus den Sprachinseln selbst. Zu erwähnen ist beispielsweise Johann Andreas Schmeller, der Sprachinseln in Venetien (wie auch dialektale Varietäten Südti-

\footnotetext{
${ }^{5}$ Georg Wenker and seine Nachfolger der Marburger Schule bezogen in ihre Arbeiten Ergebnisse der Sprachinselforschung mit ein. Erinnert sei hier nur an die engen Kontakte der wolgadeutschen Dialektologen um Georg Dinges mit Ferdinand Wrede (sowie auch Walter Ziesemer), die Dinges in den stalinistischen Prozessen wegen "Nationalismus", "konterrevolutionärer Aktivitäten" und "Spionage" zum Verhängnis wurden; cf. Rosenberg (ersch. demn.).

${ }^{6}$ Eine Reihe von Dialektologen (und zahlreiche Philologen) in der Tradition der Leipziger Schule legten - nach dem Vorbild der prototypischen "Ortsgrammatik" von Jost Winteler 1876 - vor allem um die Jahrhundertwende Ortsgrammatiken von Sprachinselvarietäten vor: Die erste "Ortsgrammatik" einer Sprachinselmundart (Zips) erstellte V. Lumtzer (1894): "Die Leibitzer Mundart". In: Paul und Braune, Beiträge zur Geschichte der deutschen Sprache und Literatur. Bis zum Ersten Weltkrieg entstand eine Reihe ähnlicher Arbeiten über den Großteil der Sprachinseln in Ungarn und mehrere Studien über Sprachinseln in den Alpen, darunter als bekannteste Bohnenberger (1913).

${ }^{7}$ Theodor Frings und seine Mitstreiter legten vor allem in der Zwischenkriegszeit interdisziplinäre Studien (unter Einbeziehung geschichtswissenschaftlicher und völkerkundlicher Forschungen) zu den Sprachinseln im Gebiet der deutschen Ostkolonisation vor.
} 
rols) in sein berühmtes Bairisches Wörterbuch aufnahm. ${ }^{8}$ Auch Jakob Grimm förderte Wortschatzsammlungen in Sprachinseln.

Zunächst richtete sich das Interesse auf die "alten" Sprachinseln, die in engerer räumlicher Beziehung zum geschlossenen deutschen Sprachraum standen, etwa in Siebenbürgen, der Zips, Venetien. ${ }^{9}$ Im 19. Jahrhundert wurden Gottschee, Temesvar, das Banat einbezogen, und ab 1870 sogar Sprachinseln in Pennsylvania $^{10}$ und Australien. Ab der Jahrhundertwende wurden zunehmend auch die jüngeren Sprachinseln zum Gegenstand dialektologischer Studien,u. a. auch von Forschern aus den Sprachinseln selbst. Nach dem Ersten Weltkrieg gerieten die Deutschen in Russland ins Blickfeld des Interesses, zuerst in den großen Städten, dann in den Kolonien im Schwarzmeergebiet und um Petersburg (Schirmunski, Ström) sowie an der Wolga (Dinges, Dulson).

Zwischen den Weltkriegen stieg die Zahl von Sprachinselstudien merklich an. Zum Teil verdankt sich dies dem Umstand, dass die deutschen Sprachinseln als in ihrer Existenz bedroht angesehen wurden. Das generell aus der Dialektologie bekannte Motiv, Sprachvarietäten angesichts eines angenommenen unvermeidlichen Untergangs zu archivieren, zeigte sich auch in der Sprachinselforschung. Diese veränderte Motivation reflektierte eine Entwicklung in den Sprachgebrauchsstrukturen zahlreicher Sprachinseln:

In Zeiten der Modernisierung, der Staatenbildung und administrativer Vereinheitlichungsbestrebungen gerieten diese Sprachinseln in intensiveren Kontakt mit den Mehrheitsgesellschaften. Dies verursachte zunächst eine "äußere" Diglossie der Kolonien: Die Mehrheitssprache wurde zuerst in der Verwaltung (im Verkehr mit Behörden außerhalb der Kolonien), im Handel und in der (höheren) Bildung eingeführt, sie blieb jedoch auf eine schmale Schicht von in diesen Bereichen Tätigen beschränkt, während der innere Verkehr der Sprachinseln fast ausschließlich auf Deutsch geschah.

\footnotetext{
${ }^{8}$ Die bairische Varietät der "Sette Communi" in Norditalien wurde von manchen Autoren als ältester deutscher Dialekt bezeichnet (Hornung 1994: 20). Die Varietät hat eine Anzahl alt- und mittelhochdeutscher Merkmale bewahrt,u. a. einen alten Konsonantismus; das Präteritum, das in anderen bairischen Varietäten bereits 400 Jahre zuvor verschwunden ist, existiert hier noch ebenso wie der Genitiv und einige weitere Merkmale.

${ }^{9}$ Erste Beschreibungen von Sprachinseln gehen zurück bis ins 16. Jahrhundert (Siebenbürgen) und ins 17. Jahrhundert (Zips) (cf. Kuhn 1934: 76f.). Der Begriff "Sprachinsel" wurde 1847 zum erstenmal für eine slawischsprachige Gemeinschaft in deutschsprachiger Umgebung in der Nähe von Königsberg, Ostpreußen, verwendet (cf. Mattheier 1996: 812).

${ }^{10}$ Die ersten Studien zu Sprachinseln, weit entfernt vom geschlossenen deutschen Sprachgebiet, wurden zum "Pennsylvania Dutch" durchgeführt. Zu ihnen gehörten die Studien deutsch-amerikanischer Forscher wie S. S. Haldemann 1872 oder die Wörterbücher von A. R. Horne 1875 und J. C. Lins 1887.
} 
Seit dem späten 19. Jahrhundert sahen sich zahlreiche Sprachinseln jedoch einem zunehmenden Eingliederungsdruck nationaler und lokaler Behörden ausgesetzt ${ }^{11}$ - zuerst in Ungarn, gegen Ende des Jahrhunderts auch in Russland, in der Zwischenkriegszeit in Polen und der Tschechoslowakei, in der Folge des Ersten Weltkriegs ebenfalls in Nordamerika und seit den 1940ern schließlich auch in Südamerika.

Mit wachsendem Kontakt und steigender Mobilität wurde die Diglossie zu einer inneren Erscheinung: Die Autonomie der deutschsprachigen Siedlungen wurde Schritt für Schritt beseitigt, lokale Eliten begannen sich zu assimilieren. Der Zweite Weltkrieg brachte schließlich für die meisten deutschen Sprachinseln in Ost- und Ostmitteleuropa die Flucht, Vertreibung und Aussiedlung ihrer Bewohner nach Deutschland.

Heute noch existierende deutsche Sprachinseln befinden sich nahezu überall auf der Welt im Niedergang: Eine stabile (dialektale, gelegentlich auch standardsprachliche) Sprachkompetenz und ein entsprechender Sprachgebrauch des Deutschen sind fast nur noch bei den Ältesten anzutreffen; die in den mittleren Generationen verbreitete Zweisprachigkeit erweist sich als Übergangserscheinung zu neuer Einsprachigkeit der jüngeren Generationen in der Mehrheitssprache.

Unter dieser Gesamtentwicklung deutscher Sprachinseln verbirgt sich allerdings eine Vielfalt sehr unterschiedlicher innerer und äußerer Bedingungen: Unterschiede in der Siedlungskontinuität, in der ethnischen, sprachlichen, religiösen Struktur der Sprachgemeinschaft und in den Beziehungen zur Mehrheitsgesellschaft.

Sprachinseln zeigen sich in Geschichte und Gegenwart als in ihrer inneren Struktur wie in ihrem Verhältnis zur Mehrheitsgesellschaft komplexer Gegenstand:

Sprachinseln sind von Siedlern unterschiedlicher regionaler Herkunft und verschiedener dialektaler Varietäten in unterschiedlichen Migrationsperioden unter verschiedenartigen Bedingungen gegründet worden.

Manche Sprachinseln sind Gemeinschaften mit einer eingeschränkten sozialen und sprachlichen Struktur: Sie haben über Jahrzehnte oder Jahrhunderte ihre Eigenständigkeit bewahren können, weil sie in ethnischem, kulturellem, religiösem, sozialem und/oder wirtschaftlichem Abstand zur Mehrheitsgesellschaft standen und Sonderbedingungen unterlagen, zu denen finanzielle "Privilegien" und Autonomierechte in administrativer, bildungspolitischer und sprachlicher Hinsicht gehörten.

${ }^{11}$ Zur "Entgermanisierung" in Ost- und Ostmitteleuropa im Gefolge von nationalen Bewegungen und Staatenbildung cf. van der Plank (1978). 
Diese auf Absonderung beruhende Struktur resultierte häufig in einem stark nach innen gerichteten Kommunikationsnetzwerk und einem eingeschränkten sprachlichen Varietätensystem. In Abhängigkeit von Siedlungsdauer und -kontinuität, von der Größe der Sprachinsel und ihrer inneren Zusammensetzung, der Distanz zur Umgebungsbevölkerung und Eingriffen von außen zeigen die deutschen Sprachinseln eine Abfolge von Entwicklungsstufen in den sprachlichen Varietätensystemen: von in einem Ort nebeneinander bestehenden lokalen Varietäten (wie in Sibirien und Kasachstan) über mehr oder weniger einheitliche Misch- und Ausgleichsvarietäten (wie in der Wolgaregion oder bei den Mennonitenkolonien im Chaco/Paraguay), über regionale Verkehrsvarietäten und Koines (wie das "Hunsrückische" in Südbrasilien oder die wolhyniendeutsche Varietät) bis zu städtischen Varietäten mit großräumiger Verbreitung (wie in Hermannstadt/Sibiu, Siebenbürgen/Rumänien).

Heute sind die deutschen Sprachinseln nahezu ausnahmslos einem intensiven Sprachkontakt ausgesetzt. Mit wachsendem Sprachkontakt zur Mehrheitsgesellschaft wächst die sprachliche Konvergenz, und mit ihr verändern sich die Aufgaben der Sprachinselforschung.

Homogenität und "Reinheit" der Sprachinselvarietäten sind heute eher die Ausnahme als die Regel. In modernen und mobilen Gesellschaften wird die Sprachinsel- und Sprachminderheitenforschung sich vom Prototyp einer abgelegenen, durch Naturraum, ethnische Zugehörigkeit, Sozialstruktur und Kultur von der Umgebung isolierten Gemeinschaft von "Hintersassen" verabschieden müssen, wenn sie nicht selbst zu einer Art von "Baströckchen-Forschung" verkommen will.

In dem Maße, wie die Sprachinseln aus der Abgeschiedenheit früherer Jahrhunderte heraustreten und in Austausch mit der Mehrheitsgesellschaft geraten, wird Sprachinselforschung sich mit der Erforschung sprachlicher Konvergenz oder Divergenz zu beschäftigen haben: Aus soziolinguistischer Perspektive hat sie die Frage zu beantworten, ob, wie und warum eine mehr oder weniger selbständige Sprachgemeinschaft ihre Distinktivität ${ }^{12}$ gegenüber einer anderssprachigen Umgebung wahren kann - oder ob, wie und warum sprachliche Konvergenz zum sukzessiven Verlust dieser Distinktivität und zur "Überflutung" der Sprachinsel führt.

Zunächst scheint dies auf den Bereich interlingualer Konvergenz zu verweisen. Da Sprachinseln jedoch häufig Gemeinschaften sind, die in sich mehrere dialektale Varietäten beherbergen, richtete sich das Forschungsinteresse auch auf ihre Rolle als "Schneller Brüter" kleinräumiger, aber intensiver intralingualer

${ }^{12}$ Mattheier (1996: 817) definiert dies als den Hauptgegenstand einer soziolinguistischen Sprachinselforschung. 
Konvergenz. Interlinguale und intralinguale Konvergenz sind kontaktbedingte sprachliche Entwicklungen. Sie treten mit "innerem" Sprachwandel wie "Vektoren" in ein Wechselverhältnis, können einander entgegenlaufen, sich neutralisieren oder einen gemeinsamen "Resonanzfall" bilden.

Dieses Wechselverhältnis anhand einiger Beobachtungen in deutschen Sprachinseln unter sehr verschiedenen Sprachkontaktbedingungen $\mathrm{zu}$ beleuchten, ist Gegenstand des folgenden Kapitels.

\section{Sprachliche Konvergenz in deutschen Sprachinseln}

Sprachinselforschung kann aus vier Gründen einen wertvollen Beitrag zur Untersuchung sprachlicher Konvergenz leisten:

1 Sprachinselgemeinschaften verfügen oft über mehrere dialektale Varietäten, die sprachlichen Ausgleichsprozessen ausgesetzt sind.

2 Eine überdachende Standardsprache des eigenen Diasystems ist in der Sprachinsel oft nicht präsent und setzt Sprachwandel und Sprachmischung daher kein Hindernis entgegen.

3 Sprachinselvarietäten sind intensivem Sprachkontakt ausgesetzt. Der Vergleich der sprachlichen Entwicklungen in Sprachinseln im Kontakt mit unterschiedlichen Sprachen gibt Aufschluss darüber, inwieweit Sprachwandel durch interne oder externe Faktoren (oder beides) induziert ist.

4 Da die Distinktivität der Sprachgemeinschaft über die Aufrechterhaltung oder Assimilation der Sprachinseln entscheidet, ist Sprachinselforschung notwendig interdisziplinär angelegt und vereint soziolinguistische, historische, ethnologische und andere Methoden.

Die Beschäftigung mit sprachlicher Konvergenz verlangt jedoch zunächst einmal einige Unterscheidungen, was der Gegenstand von "Konvergenz" sein soll:

Zunächst ist zu klären: Was konvergiert womit? Handelt es sich um eine dialektale Varietät, die mit einer anderen Varietät desselben Diasystems konvergiert (einer anderen dialektalen Varietät, einer Standardvarietät, einem Regiolekt)? Oder konvergiert diese mit einer Kontaktsprache (eines anderen Diasystems)?

Was ist das Ergebnis der Konvergenz? Eine einzige Varietät als Resultat eines Ausgleichsprozesses oder konvergierende Strukturen von nach wie vor selbständigen Varietäten - oder weder das eine noch das andere, sondern eine Art von "Koine", d.h. eine "höhere" Sprachlage im sprachliche Variationssystem der Sprachgemeinschaft?

Wenn intralinguale oder auch interlinguale Konvergenzphänomene feststellbar sind, treten sie auch in anderen deutschen Varietäten auf? Wenn dies der Fall ist, ist dies ein Anzeichen eines rein inneren, etwa typologischen, Sprachwandels, dem alle deutschen Varietäten früher oder später ausgesetzt sind: eine Art von 
polyzentrischer "Konvergenz" im Sinne einer Angleichung der Varietäten von verschiedenen Entwicklungsstufen aus? ${ }^{13}$

Wenn wir also die aufgeworfenen Fragen auf systematische Weise beantworten wollen, werden wir das Problem der Konvergenz in drei elementare Probleme aufbrechen müssen:

\subsection{Konvergenz als Folge des Varietätenkontakts}

Dialektkonvergenz ist der klassische Gegenstand der russlanddeutschen Sprachinselforschung.

Deutsche Siedlungen wurden seit 1763 an der Wolga, dann im Schwarzmeergebiet, im Kaukasus und später in Sibirien und Mittelasien gegründet. Diese Kolonien zeigten eine einzigartige Vielfalt von dialektalen Varietäten, die aus verschiedenen deutschen Herkunftsregionen in die Kolonien gelangten und sich dort mischten. In vielen Siedlungen wurden zunächst mehrere dialektale Varietäten gesprochen, die aufgrund der räumlich separaten Lage und des sozialen, ökonomischen, sozialen und religiösen Abstands von der umgebenden Bevölkerung über längere Zeit überdauerten.

Von Beginn an setzte ein sprachlicher Ausgleich ein, der in mehrere Konvergenzphasen eingeteilt werden kann:

Der sprachliche Ausgleich während der ersten hundert Jahre ließ aus der extremen Vielfalt von Dialekten weitgehend einheitliche dörfliche Mischvarietäten entstehen, führte jedoch nicht zur Herausbildung eines einheitlichen "Russlanddeutschs" oder großräumiger "Umgangssprachen". Die Deportationen und Verfolgungen - spätestens seit 1938/41 - stellten die entscheidende Zäsur in der Sprachentwicklung der Russlanddeutschen dar: Die Auflösung der geschlossenen deutschen Sprachinseln, der Abbruch der Siedlungskontinuität und die Stigmatisierung der "Sprache der Faschisten" brachen den sprachlichen Ausgleich ab und setzten die deutschen Siedlungen einem wachsenden Einfluss des Russischen aus.

\footnotetext{
${ }^{13}$ Coseriu (1975) nennt solche Phänomene ebenfalls "Konvergenz": "sprachliche Konvergenzen, d.h. voneinander unabhängige Parallelentwicklungen, die in historisch verwandten Sprachen offenbar auftreten" (Coseriu 1975: 134). Als Ergebnis "typologischer" Konvergenz bezeichnet erz. B. die "funktionelle Kohärenz" aller romanischer Sprachen (außer dem Französischen und Okzitanischen) nach der Regel: "interner" (paradigmatischer) Ausdruck "interner", nicht-relationeller Funktionen (wie Genus und Numerus); "externer" (syntagmatischer) Ausdruck "externer", relationeller Funktionen (wie Kasus oder Steiegerung des Adjektivs) (cf. Coseriu 1975: 142).
} 
Die Deportationen und anschließenden Weiterwanderungen nach Aufhebung der Sondersiedlungen der "Kommandantura" verstärkten die sprachliche Heterogenität in den neuen Siedlungsgebieten der Russlanddeutschen.

Mit der Bildung von Mischsiedlungen in Sibirien und Mittelasien wurde der sprachliche Ausgleichsprozess erneut in Gang gesetzt. Dieser fand nun jedoch unter den völlig veränderten Verhältnissen eines intensiven Sprachkontakts zum Russischen und einer inneren Diglossie statt. Die "Sowjetisierung" der Gesellschaft, eine gewisse Modernisierung und einheitliche Organisation des Staatsapparats sowie bescheidene Liberalisierungen gegenüber den Russlanddeutschen förderten die Assimilation.

Mit der Gründung zentraler Kolchosen und schließlich der Bildung von "Zentraldörfern" aus aufgelösten kleineren Siedlungen (seit den 1970ern) wurde die dritte und letzte Phase des sprachlichen Ausgleichs eingeleitet. Sprachliche Konvergenzphänomene sind heute in diesen Orten "in vivo" beobachtbar, jedoch deutlich überprägt von einem rapiden Sprachwechsel zum Russischen, insbesondere unter den jüngeren Generationen.

Schon Viktor Schirmunski (1930: 113f.) hatte den sprachlichen Ausgleich und die Dialektmischung in den russlanddeutschen Varietäten als ein "großangelegtes sprachgeschichtliches Experiment" und als "sprachwissenschaftliches Laboratorium" bezeichnete, "in dem wir an der Hand geschichtlicher Zeugnisse in einer kurzen Zeitspanne von 100 bis 150 Jahren Entwicklungen verfolgen können, die sich im Mutterlande in mehreren Jahrhunderten abgespielt haben müssen". ${ }^{14}$

Als ein interessanter Fall sprachlicher Konvergenz, der immer wieder das Interesse namhafter Dialektologen auf sich gezogen hat (cf.u. a. Schirmunski 1930, Jedig 1966), können die niederdeutschen Varietäten der Russlandmennoniten angesehen werden, die heute in Westsibirien und der Orenburger Region leben. Innerhalb ihrer stark nach innen gerichteten Kommunikationsnetzwerke fand bei den Mennoniten bereits in der Ukraine ein frühzeitiger und weitreichender Ausgleichsprozess statt, der zwei Varietäten entstehen ließ: die ältere ChortitzaVarietät und die - gemeinhin als prestigereicher angesehene - MolotschnaVarietät.

Lange Zeit war angenommen worden, dass im Kontakt dieser beiden Varietäten eine "aufwärtsgerichtete" Konvergenz stattfände, die die Merkmale der Chortitza-Varietät durch Merkmale der Molotschna-Varietät ersetze: Dies galt z. B.

\footnotetext{
${ }^{14}$ Selbst wenn sich dies als Mythos erweisen könnte, der einer Überschätzung der Koinesierung in den Schwarzmeerkolonien entsprang, war Schirmunskis Orientierung auf die Erforschung des Dialektausgleichs - statt auf die "Heimatbestimmung" der Sprachinselvarietäten - richtungsweisend.
} 
für die Verdrängung des [y:] der Chortitza-Varietät durch das [u:] der Molotschna-Varietät, wie etwa in [fry:] - [fru:] (hd. 'Frau').

Viktor Schirmunski entwickelte anhand der Konvergenzphänomene in den russlanddeutschen Varietäten seine berühmte Theorie der "primären" und "sekundären" Dialektmerkmale: Nach Schirmunski (1930) werden im Kontakt sprachlicher Varietäten die "auffälligsten" Merkmale der dominanten Mundart beibehalten, die der untergeordneten Mundart dagegen aufgegeben. Weniger "auffällige" Merkmale beider Varietäten mischen sich. Die Richtung des sprachlichen Ausgleichs sei mit dem Standarddeutschen (oder einer Koine) gegeben.

"Wir bezeichnen im weiteren die charakteristischen, d.h. am stärksten auffallenden Abweichungen einer Mundart gegenüber der Schriftsprache (oder anderen Mundarten) als primäre Merkmale, die weniger auffallenden Abweichungen als sekundäre Merkmale." (Schirmunski 1930: 118).

Da jedoch das Standarddeutsche als Kriterium der "Auffälligkeit" und damit der sprachlichen Konvergenz in den russlanddeutschen Siedlungen über weite Strecken kaum eine Rolle spielte, blieb der Erklärungswert dieser Interpretation zweifelhaft. ${ }^{15}$ Andererseits ließ sich im Schwarzmeergebiet in Ausgleichsvarietäten hessischen und schwäbischen Ursprungs durchaus eine überwiegende Konvergenz in Richtung "fränkischer" oder standarddeutscher Merkmale nachweisen.

In einer Pilotstudie unter den Mennoniten der Orenburger Region hat Nyman (1997) eine komplexere Struktur des sprachlichen Ausgleichs beobachtet: ${ }^{16}$ Einige Merkmale folgen dem Molotschna-Muster, andere dem Chortitza-Muster, und wieder andere zeigen überhaupt keinen Ausgleich.

Im Ausgleich zwischen [u:] - [y:] - zum Beispiel in [u:t] - [y:t] (hd. 'aus') - hat sich das Chortitza-Merkmal [y:] über sämtliche 22 Mennonitendörfer verbreitet. ${ }^{17}$ Dies ist mit der Annahme einer sukzessiven Verdrängung der Chortitza-Merkmale schwer vereinbar, ebenso wenig wie mit einer Ausgleichsrichtung der primären Merkmale, die am Standarddeutschen (oder einer Koine) als Auffälligkeitsmaßstab orientiert ist: Das Chortitza-Merkmal [y:] ist

${ }^{15}$ Dies umso mehr, als die von Schirmunski (1930) ersatzweise ins Feld geführte "fränkische Gemeinsprache" (Koine) im Schwarzmeergebiet weniger eine tatsächlich verwendete Ausgleichsvarietät als eine gemeinsame Merkmalsmenge von Ortsvarietäten darstellt.

${ }^{16}$ Nyman (1997) untersuchte die Richtung des sprachlichen Ausgleichs in den um 1900 besiedelten und 1997 noch bestehenden 22 Mennonitendörfern der Orenburger Region, indem er bei Gewährsleuten sogenannte "Distinktionswörter" erhob, an denen eine Unterscheidung von Chortitza-Varietät und Molotschna-Varietät erfolgen sollte.

17 Ähnliche Erscheinungen aus anderen Mennonitensiedlungen berichten Berend/Jedig (1991: 177). 
sicher vom standarddeutschen [au] weiter entfernt als das Molotschna-Merkmal [u:]. Dieser Sprachausgleich, der wie ein "change from below" anmutet, verdankt sich möglicherweise dem numerischen Übergewichts der Sprecher der Chortitza-Varietät, die die Mehrzahl der Dörfer besiedelten, oder es handelt sich um ein Traditionssymbol, eine einstellungsbedingte Gegenentwicklung zu dem starken Anpassungsdruck der russischen Außenwelt.

Die Verteilung des Molotschna-Lexems knout und des Chortitza-Lexems strank (für hd. 'Seil') zeigt - wie einige weitere Merkmale - eine strikte Trennung zwischen Molotschna- und Chortitza-Varietät.

Die [-n]-Apokope der Molotschna-Varietät setzt sich hingegen im Bereich des nominalen Pluralmarkers und des Infinitivsuffixes durch. Die Apokope hat sich gegen die Chortitza- Merkmale, die mit den standarddeutschen gleichlauten, durchgesetzt, allerdings nur in den Dörfern in der Umgebung der MolotschnaGruppe. Diese Gruppe von Dörfern bildet eine neue subregionale Einheit und wird als "Unjadarpa" ('Unterdörfer') im Gegensatz zur Restgruppe der "Bowadarpa" ('Oberdörfer') bezeichnet.

Dania Asfandiarova (1999) hat in einer Studie im Gebiet von Ufa, Baschkortostan, interessante Konvergenzphänomene untersucht. ${ }^{18}$ Im Vokalsystem der Sprecher (der ältesten Generation) zeigt sich eine komplexe und überraschende Struktur. In den drei "lutherischen" Dörfern überwiegen rheinfränkische Merkmale, das vierte, ein "katholisches" Dorf, zeigt jedoch frappierende Unterschiede: Der sprachliche Ausgleich ist in den Vorderzungenvokalen sowie den geschlossenen Hinterzungenvokalen zwischen den Varietäten nahezu vollständig vollzogen, jedoch nicht für (mhd.) langes und kurzes $a$ and das Diphthongsystem. Hier verwenden die "katholischen" Sprecher ein verdumpftes $a$, ein halboffenes oder sogar geschlossenes $o$, wie etwa in [gro:s] 'gras' oder [vo:re] 'waren'. Diese gelten als Kennzeichen der "katholischen" Varietät, die im "katholischen" Dorf und von den "Katholischen" in den anderen Dörfern gesprochen wird. Diese Sprecher haben offenbar einige emblematische Merkmale ihrer Varietät bewahrt, die sie als "Achterisch" bezeichnen (die Nummer

\footnotetext{
${ }^{18}$ Dania Asfandiarova hat den Dialektausgleich in der Sprachinsel Prishib/Alekseevka in vier Dörfern untersucht, die von ehemals elf Siedlungen zur Zeit der Besiedlung um 1900 noch übrig geblieben sind. Jedes Dorf besaß ursprünglich seine eigene Varietät; die Aufgabe kleiner Siedlungen und die Bildung des Zentraldorfs Prishib hat den sprachliche Ausgleich seit den frühen 1980ern stark beschleunigt.

Harald Weydt und ich waren 1990 mit dem großen Mennonitensprachforscher Heinrich Klassen in diesen Dörfern - nach einer abenteuerlichen Fahrt bei $-49^{\circ} \mathrm{C}$ ! Diese Kälte, der Wodka oder die Polka mit beleibten russlanddeutschen Damen ließen wohl in Harald Weydt den Traum reifen, in Prishib einmal den Sommerurlaub zu verbringen. Nun ist die Zeit dazu vielleicht vorhanden!
} 
ihres Ortes zur Zeit der Besiedlung), im Unterschied zum "Sechserisch", der Varietät der "lutherischen" Mehrheit der anderen Dörfer - dies, obwohl die Varietäten ansonsten vollständig ausgeglichen sind.

Die Konvergenz scheint in diesen Fällen ein höchst komplexer, selektiver, von vielerlei Faktoren beeinflusster Vorgang zu sein, der alle denkbaren Richtungen annehmen und unter Umständen von Merkmal zu Merkmal differieren kann.

Wie Andreas Dulson in den 1930ern anhand seiner Studien in dialektal extrem heterogenen wolgadeutschen Dörfern festgestellt hat, scheint Schirmunskis Kriterium der "primären" und "sekundären" Merkmale lediglich einen von mehreren Einflussfaktoren der Konvergenz anzugeben, der jedoch häufig mit anderen Faktoren in Verbindung tritt. Dulson (1941: 85) schlug sieben Einflussfaktoren vor: die sprachliche Norm der Standardsprache oder einer regionalen Verkehrsvarieät (wenn jeweils vorhanden), die Struktur der in Ausgleich tretenden dialektalen Varietäten, das soziale Prestige der Sprechergruppen, die Spracheinstellungen der Sprecher zu den Varietätenmerkmalen, den Grad der sprachlichen Heterogenität innerhalb der Sprechergemeinschaft und die inneren Entwicklungstendenzen des sprachlichen Wandels in den deutschen Varietäten.

Die Feststellung, ob ein dialektales Merkmal als "primär" oder "sekundär" bezeichnet werden kann, hängt von ihrer Auffälligkeit im Sprachwertsystem der Sprecher ab. Was als "auffällig" gilt, stimmt nicht notwendig mit linguistischen Kriterien des sprachlichen Abstands überein, ist nicht unbedingt eine Frage der Systematizität, des Phonemstatus oder der Abweichung von der Standardsprache, sondern des "Monitorings" der Sprecher. "Auffälligkeit" ist paradigmatisch, auf den Einzelfall bezogen, geordnet nach Markiertheitsregeln und von den Spracheinstellungen der Sprecher abhängig.

Wenn sich etwa kompakte Sprechergruppen gegenüberstehen, können die in Kontakt tretenden Varietäten oder einzelne Merkmale, die als Marker zur Abgrenzung der Sprechergemeinschaften verwendet werden, über lange Zeit erhalten bleiben. Ob solche Gruppen als "kompakt" anzusehen sind, beurteilt sich nach der numerischen Stärke und Distinktivität der Gruppen, der Stabilität der Gruppennormen (in sprachlicher wie kultureller Hinsicht), der Festigkeit von Einstellungen zur Eigen- und Fremdgruppe und der Neigung zur sprachlichen "Akkomodation" (zu Konvergenz- oder Divergenzverhalten im Sinne von Niedzielski/Giles 1996).

Je höher der Grad an Heterogenität innerhalb einer Sprechergemeinschaft ist, desto geringer wird der Effekt sprachlicher Normen sein - und desto rascher wird der sprachliche Wandel ablaufen - bis zu dem Punkt, an dem "rein" sprachinterne Entwicklungsgesetze am Werk zu sein scheinen. Dann - so Dulson (1941: 93) - handle es sich weniger um Konvergenz oder Divergenz von 
Varietäten als um einen "Kampf, der zwischen den einzelnen Spracherscheinungen getrennt gefochten wird".

\subsection{Konvergenz als Folge des Sprachkontakts}

Sprachinseln sind sprachliche Gemeinschaften in fremder Umgebung, d.h. in der Regel isoliert vom geschlossenen Binnensprachraum: Sie gehen in ihrer Entwicklung eigene Wege, manchmal stimmt ihre Entwicklungsrichtung mit der der Herkunftsvarietäten überein, manchmal läuft sie ihr zuwider. Wenn keine Übereinstimmung vorliegt - woran könnte das liegen?

Um den Einfluss der Kontaktsprache zu beurteilen, bietet sich eine vergleichende Methode in der Sprachinselforschung an: Wenn wir die unterschiedlichen Entwicklungen in den deutschen Sprachinselvarietäten im Kontakt mit der russischen Sprache, dem amerikanischen Englisch (als germanischer Sprache) und dem brasilianischen Portugiesisch (als romanischer Sprache) vergleichen, lassen sich sehr viel verlässlichere Aussagen über das Gewicht interner und externer Einflüsse treffen.

Dies sei anhand der folgenden Beispiele expliziert:

Die deutschsprachigen Siedler im südbrasilianischen Bundesstaat Rio Grande do Sul sind von ihrer historischen Entwicklung - auch wenn dies kurios anmutet durchaus den Russlanddeutschen vergleichbar:

Beide Länder haben eine starke deutschsprachige Minderheit. Brasilien liegt mit seinen ca. eine Million Deutschsprachigen auf dem zweiten Platz hinter der ehemaligen Sowjetunion (mit ca. zwei Millionen).

Deutsche haben in Brasilien wie in Russland in weitgehend isolierten "Kolonien" gelebt.

Auch in Brasilien verwendeten die Siedler vorwiegend dialektale Varietäten in der alltäglichen Kommunikation; Konvergenz zwischen diesen dialektalen Varietäten war folglich ein Wesensmerkmal der Kolonien. Dies sind dialektale Varietäten sehr unterschiedlicher Herkunft: vom dominanten "Hunsrück" (mit rhein- und moselfränkischen Merkmalen) über schwäbische und wolgadeutsche Varietäten bis zum niederdeutschen Westfälisch und "Pomerano". Die deutsche Standardsprache war zeitweise in der Schule unterrichtet worden, jedoch wurden unter der nationalistischen Regierung Getulio Vargas' in den 1940ern sämtliche schulischen und sonstigen Minderheitenrechte aufgehoben. Seitdem setzte eine "Redialektalisierung" ein.

Im Unterschied zu den Russlanddeutschen entwickelte sich mit dem Hunsrückischen eine überregionale Varietät, was in Russland nie der Fall war. Offenbar trugen die zahlenmäßige Dominanz der Hunsrück-Sprecher (über 50\%) sowie 
ein dichteres Kommunikationsnetzwerk zu einer solchen Konvergenz bei. Allerdings ging diese nicht bis zu einem völligen Ausgleich. Das Hunsrückische ist als Dialektkontinuum anzusehen, in dem rheinfränkische Merkmale mit höherem Prestige und moselfränkische mit geringerem Prestige (z.B. dat/wat) existieren. Lokale Basisvarietäten sind als "familetos" erhalten (Varietäten von einzelnen "Schneisen", Siedlungsreihen, die ursprünglich aus Familienverbänden bestanden; cf. Altenhofen 1996: 27).

Es zeigen sich neben einigen Ähnlichkeiten interessanterweise eine Reihe von unterschiedlichen Entwicklungen zwischen den deutschen Sprachinseln Brasiliens und Russlands, obwohl die Herkunftsvarietäten partiell die gleichen Merkmale trugen:

Die Sonorisierung hat sich in beiden Gruppen ausgebreitet (die Stimmhaftigkeit von auf den Wortakzent folgenden Konsonanten in intervokalischer Position und zwischen Vokalen und Sonoranten: drogge hd. 'trocken'; cf. Altenhofen 1996: Karte 29).

Andererseits haben sich die Nasalierung und der Rhotazismus unterschiedlich entwickelt:

Die Nasalierung ([tsã:] hd. 'Zahn') ist ein verbreitetes Merkmal unter den wolgadeutschen Varietäten (cf. Wolgadeutscher Sprachatlas 1996: Karte 143), nicht aber unter den Hunsrück-Sprechern, obwohl die dialektale Basis diesbezüglich ähnlich war und das brasilianische Portugiesisch mehrere nasalierte Vokale besitzt.

Der Rhotazismus ([sore], hd. 'sagten'; cf. Altenhofen 1996: Karte 56) ist in Brasilien in vielen Wortformen vorhanden, in Russland jedoch nur unter den Sprechern einer oberhessischen Varietät. Dies mag sich dem Umstand verdanken, dass die Wolgadeutschen mindestens 60 Jahre früher auswanderten als die Brasiliendeutschen, zu einer Zeit, als sich dieses Merkmal im deutschen Sprachgebiet gerade erst ausbreitete (cf. Schirmunski 1962: 317ff.; Dulson 1941: 96).

Externer sprachlicher Einfluss spielt in diesen Fällen offensichtlich eine geringe oder gar keine Rolle. Selbstverständlich gibt es andere Erscheinungen, bei denen sprachliche Interferenz eine erheblich größere Bedeutung besitzt: Die geringe (und schwankende) Verwendung des Artikels unter jüngeren Russlanddeutschen ist sicherlich primär auf den Einfluss des Russischen zurückzuführen, wie Dorothea Nöth (1994) herausfand: Russlanddeutsche, die auch nur eine rudimentäre (z. T. lediglich rezeptive) Sprachkompetenz einer deutschen Varietät besaßen, erreichten im Artikelgebrauch deutlich bessere Ergebnisse als Probanden ohne eine solche Kompetenz (cf. Nöth 1994: 127). Sprecher brasiliendeutscher Varietäten velarisieren andererseits den Lateral /1/, was mit hoher Wahrscheinlichkeit auf portugiesische Interferenz zurückgeht. 
Eine Entwicklung, die häufig zum Gegenstand von Untersuchungen erhoben wurde, ist die Reduktion der Kasusmorphologie unter Deutschsprachigen in Russland (cf. Jedig 1966), in Brasilien (cf. Altenhofen 1996: 254ff.), in Texas (cf. Salmons 1994: 60), Pennsylvania (cf. Louden 1994: 84; Huffines 1989: 216ff; 1994: 50f.) und unter Wolgadeutschen in Kansas (cf. Keel 1994: 98) in den USA $^{19}$ sowie in anderen Ländern.

Externe Effekte sind sicherlich denkbar im Kontakt der deutschen Sprachinselvarietäten mit germanischen und romanischen Sprachen (cf. Louden 1994: 90). Andererseits lässt sich verstärkter Kasusverlust unter Mennoniten- und AmishGruppen gerade bei sogenannten "Sectarians" beobachten, die ihre Varietäten durchgängig im alltäglichen Gebrauch verwenden, während sogen. "NonSectarians" mit intensivem Sprachkontakt zum Englischen einen deutlich geringeren Kasusabbau zeigen.

Für den Kasussynkretismus unter Russlanddeutschen wird der Einfluss des Russischen nicht in Anspruch genommen werden können. Das Russische besitzt sechs Kasus, die in der Nominal-, Pronominal- und Adjektivflexion konsequent verwendet werden. Russische Interferenz wäre eher geeignet, das Flexionssystem der deutschen Sprachinselvarietäten zu stützen und den Kasusabbau zu verlangsamen. Dies ist jedoch nicht der Fall. Im Gegenteil ist der Kasussynkretismus gerade bei den russlanddeutschen Gruppen am stärksten, die den größten russischen Einfluss aufweisen.

\subsection{Typologische "Konvergenz"}

Offensichtlich haben wir es im Falle des Kasussynkretismus mit einem typologischen Wandel zu tun, der in den deutschen Varietäten überall auf der Welt zu beobachten ist. Der Kasusverfall ist ebenso wie der Präteritumsschwund Bestandteil einer langfristigen Entwicklung von synthetischen zu analytischen sprachlichen Strukturen. Das Deutsche ist auf dieses Kriterium bezogen selbstverständlich ein "Mischtyp" (cf. Lang 1996: 12). Typologie vergleicht heute nicht Sprachen, sondern Strukturen (cf. Comrie 1996: 16). Folgerichtig argumentieren einige Forscher, dass das Deutsche keine uniforme typologische Entwicklungsrichtung aufweise. Die vorwiegende Richtung besteht jedoch zweifellos in einer Reduktion synthetischer (oder "fusionierender"; cf. Wurzel 1996: 522) Formen und einer "Externalisierung" syntaktischer Merkmale, etwa der Distribution funktionaler Merkmale auf unterschiedliche Formelemente, von denen jedes nur wenig grammatische Information trägt.

${ }^{19}$ Huffines (1989: 212ff.) fasst den Forschungsstand zum Kasussynkretismus in den USA zusammen, insbesondere zum Verlust des Dativ. 
Seit der Verlagerung des Wortakzents auf den Stammvokal unterliegt die Kasusmorphologie der deutschen Nominalflexion einem steten Reduktionsprozess. Heute ist die Nominalflexion weitgehend auf die Markierung des Genitiv beschränkt (cf. Eisenberg 1994: 362); nur das starke Deklinationsschema kennzeichnet auch den Dativ Plural (während die des Dativ Singular optional ist). Die Kasusmarkierung in der Nominalphrase basiert auf dem Prinzip des "geringsten Aufwandes": "The adjective marks the noun phrase for case, number and gender [...], if no other constituent of the noun phrase does so" (Eisenberg 1994: 367). Kasus, Numerus und Genus werden generell durch den Artikel (oder Pronomina) gekennzeichnet. Die grammatische Information rückt somit in der Phrasenstruktur zunehmend weiter nach links, d.h. weg vom Kopf der Nominalphrase zum Adjektiv oder weiter zum Artikel, in eine prädeterminative Position.

Die Reduktion der Nominalflexion in den dialektalen Varietäten des Deutschen geht jedoch weiter und tiefer. Im allgemeinen verfügt das Nominalsystem nur noch über zwei Kasus (drei Kasus in einigen schwäbischen Varietäten). Der Genitiv ist in den meisten Funktionen ersetzt durch präpositionale ("dat hüs von min brouder") oder Dativkonstruktionen (mit Possessivpronomen: "minem brouder sin hüs"). Dativ und Akkusativ sind im allgemeinen in einem Obliquenkasus zusammengefallen (mit wechselnden Formen, im Oberdeutschen und z. T. im Westmitteldeutschen gelegentlich auch in Dativform, zumeist aber in Akkusativform).

In der Nominalflexion wird der Genitiv als erstes aufgegeben, relativ stabil ist er nur in der Funktion des Possessivs, vor allem bei Eigennamen; der Dativ wird als nächstes abgebaut, nur wenige dialektale Varietäten markieren den Dativ noch am Nomen (z.B. einige westfälische Varietäten). Der letzte Vorposten der Nominalflexion ist der Akkusativ, der auch dem Obliquenkasus die Form gibt. ${ }^{20}$

${ }^{20}$ Innerhalb der Markiertheitstheorie (oder Natürlichkeitstheorie) wird von verschiedenen Autoren eine Kasushierarchie vorgeschlagen, die unterschiedlichen Graden der Markiertheit folgt: Der Nominativ wird als am geringsten markiert (d.h. am "natürlichsten") angenommen, dann folgt der Akkusativ, der geringer markiert sei als der Dativ, dieser wiederum geringer als der Genitiv (cf. Mayerthaler et al. 1998: 167). "Natürlicher" Wandel bedeutet in diesem Sinne: Stärker markierte Elemente werden in geringer markierte Elemente überführt. Dies stimmt mit der allgemeinen Richtung des Kasusabbaus überein, wenngleich das Kriterium der "Natürlichkeit" nicht vollständig klar ist.

Ursprünglich geht dies auf Roman Jakobson (1936) zurück, der am Beispiel des russische Kasussystems eine letztlich semantisch orientierte Kasuslehre ausarbeitet: Jakobson sieht darin den Nominativ als den unmarkierten, merkmallosen Kasus, der eine reine "Nennfunktion" (ebd.: 250) ausübe und dem gegenüber der Akkusativ in einer "Bezugskorrelation" (ebd.: 249) für seine Handlungsunterworfenheit markiert sei. Der Akkusativ wiederum sei unmarkiert gegenüber dem Dativ, der im Rahmen einer "Stellungskorrelation" markiert sei für seine "periphere Stellung" (ebd.: 262) als "Empfänger des Gesche- 
Die Pronominalflexion zeigt mehr Kasusunterscheidungen als die Nominalflexion, das Maskulinum mehr das Femininum und Neutrum, der Singular mehr als der Plural. Da der bestimmte und unbestimmte Artikel (insbesondere im Maskulinum und Femininum) ebenfalls auf ein Zwei-Kasus-System reduziert werden, das überdies häufig durch enklitische Formen repräsentiert wird, wird die grammatische Information weder durch Flexion des Nomens noch des Adjektivs noch des Artikels gegeben. Dies gilt für zahlreiche deutsche Sprachinselvarietäten, unabhängig von ihrer Verbindung zum binnendeutschen Sprachraum.

In der deutschen Standardsprache erfüllt die Flexionsmorphologie unterschiedliche semantische und syntaktische Funktionen: "[The] rich inflectional morphology [...] fulfils in part purely semantic functions; on the other hand, it is in part clearly motivated syntactically" (Eisenberg 1994: 374). Wenn die Kasuskategorie in dialektalen Varietäten überhaupt markiert wird, werden syntaktische oder semantische Informationen nur morphologisch ausgedrückt, um den "Objektkasus" oder Obliquenkasus vom Nominativ zu unterscheiden, das heißt um die "Unselbständigkeit des Gegenstandes" im "Bezugskasus" (Jakobson 1936: 282) zu markieren. Weitere syntaktische (wie etwa die Kongruenz zwischen Nomen und Adjektiv) oder semantische Informationen (wie die Relation zwischen direktem und indirektem Objekt) werden zunehmend durch die Wortstellung gegeben, nicht durch die Morphologie. In Präpositionalphrasen wandert die Information - gegenüber der Standardsprache - noch einen Schritt weiter nach links bis zum lexikalischen Element, der Präposition.

Der Abbau des morphologischen Systems in den deutschen Sprachinselvarietäten lässt noch einen weiteren Beleg für einen inneren, "typologischen" Wandel erkennen, dem mehr oder weniger alle deutschen Varietäten wie auch andere indoeuropäische Sprachen unterworfen sind. Während die Reduktion der Flexionsmorphologie im Nominalsystem weit fortgeschritten ist, trifft dies für

hens", von dem die Handlung unabhängig sei (ebd.: 270). Der Genitiv sei gegenüber den anderen in einer "Umfangskorrelation" (ebd.: 255) markiert für seine Umfangsbeschränkung (oder seine beschränkte Teilhabe).

Andere Autoren betonen die syntaktische Flexibilität des Akkusativs hinsichtlich seiner - durch kommunikative oder diskursive Funktionen begründeten - grammatischen Motiviertheit: Der Akkusativ wird aufgrund seiner funktionellen Belastung als resistenter angesehen (verwendbarz. B. für Passivtransformation, Topikalisierung, Ausklammerung, Reflexivkonstruktionen, Anschluss von Relativsätzen etc.). Überdies wird der Akkusativ gestützt durch Formgleichheiten mit dem Nominativ (Femininum, Neutrum, Plural). Darüber hinaus scheinen auch phonologische Faktoren die Akkusativform zu unterstützen, etwa durch die verbreitete Ersetzung des $-m$ durch $-n$ (und gelegentlich anschließende Tilgung des $-n$ ) - (ein Vorgang, der in vielen dialektalen Varietäten beobachtbar ist und sich gelegentlich auch auf das Neutrum erstreckt: in den Haus, in Haus (cf. Rosenberg 1986: 341ff. mit ca. 300 Belegen aus schriftlichen Schülerarbeiten). 
das Pronominalsystem nicht im gleichen Maße zu: Die Flexion der Personalpronomina ist deutlich "konservativer" und erst in letzter Instanz Gegenstand von Konvergenzprozessen. Im Unterschied zum Zwei-Kasus-System der Nominal-, Adjektiv- und Artikelflexion besitzt das Personalpronomen in Teilbereichen ein Drei-Kasus-System, das die Möglichkeit einer Unterscheidung von direktem und indirektem Objekt enthält oder zumindest Dativformen bewahrt.

Das Kasussystem in den Personalpronomina des Pennsylvania German sei hier stellvertretend für viele andere Sprachinselvarietäten erwähnt (siehe Tabelle am Ende). Das System gilt für die sprachlich traditionelleren Non-Sectarians, die in gewissem Sinne stärker in die Mehrheitsgesellschaft integriert sind.

Marion Lois Huffines (1989) hat Non-Sectarians und Sectarians in einer Studie mehrerer Generationen verglichen. Für die Non-Sectarians zeigt sich eine größere Stabilität der Kasusmorphologie, die z.T. noch Nominativ, Akkusativ und Dativ unterscheidet:

"The pronoun system has three cases: the nominative, accusative, and dative. Distinctively marked dative pronoun forms occur in the 1st, 2nd, and 3rd persons singular, and in the 3 rd person plural. The interrogative personal pronoun also has two case forms, the common and the dative" (Huffines 1989: 216).

In dem Maße, in dem das Pennsylvania German bei den Non-Sectarians immer weniger an die Kinder weitergegeben wird, geht insbesondere der Dativ verloren. Unter den Sectarians werden ohnehin fast ausschließlich Akkusativ- bzw. Einheitskasusformen verwendet(cf. dazu Keel 1994: 96; Louden 1994: 84).

"The nonsectarian native speakers with few exceptions use dative forms to express dative functions. [...] The speech of the nonsectarian native speakers reflects a firmly established norm for PG dative usage. The two youngest nonsectarian native speakers diverge from that norm in ways which are consonant with the linguistic performance of the first-native-English speakers. [...] The first-in-the-family native English speakers use fewer datives and more accusative and common case forms to express dative functions than do the nonsectarian native speakers. [...] The second-in-the-family native English speakers use still fewer datives and more accusative and common case forms to express dative functions. [...] The norm established by the native speakers and aspired by the first-native-English speakers has not been acquired by Group 2 [the second-native-English speakers, P.R.]; the second-native-English speakers simply do not know the PG norm. The sectarians use accusative and common case forms to express dative functions almost exclusively." (Huffines 1989: 222f.)

In anderen deutschen Sprachinselvarietäten zeigt sich ebenfalls ein partieller Wechsel vom Dativ zum Akkusativ, etwa im Texas German: Spricht er zu mich? (cf. Salmons 1994: 66). Aber selbst hier ist die Dativmarkierung im System der Personalpronomina deutlich höher als beim Artikel (viermal so hoch; cf. Salmons 1994: 64). 
Dieses Phänomen ist andererseits ebenso aus den meisten anderen germanischen und auch einigen romanischen Sprachen bekannt, wie die folgende Synopse deutlich macht: ${ }^{21}$

Das Kasussystem der Personalpronomina im Dänischen hat drei Kasus, ${ }^{22}$ während die Nominalflexion lediglich zwischen Einheitskasus und Genitiv unterscheidet. Die Formen des Obliquenkasus in der 3. Pers. gehen jedoch auf altskandinavische Dative zurück (im Plural als Demonstr. Pron. im Distal).

Die Personalpronomina im Altenglischen zeigen in den meisten Fällen ein DreiKasus-System (cf. van Kemenade 1994). Im Neuenglischen gehen die Pronomina der 3. Pers. Sing (Mask. und Fem.) und Plur. auf altenglische Dativformen zurück (die im Mittelenglischen verloren gegangen sind), während die Nominalflexion nur noch zwischen Einheitskasus und Possessiv (alten Genitiven) unterscheidet.

Das Französische hat bekanntlich nahezu jegliche Flexion verloren, wie Charles Bally (1965: 193) feststellt:

"Le français s'est débarrassé de la plupart des flexions héritées du Latin: elles ont prèsque disparu du substantif, végètent dans l'adjectif (distinction sporadique du genre et du nombre) et ne subsistent à l'état de demi-système que dans le verbe, où d'ailleurs les destinances sont constamment battues en brèche (remplacement de nous par on, abandon du passé défini, de l'imparfait du subjonctif, déclin du reste de ce mode)".

Das Kasussystem der französischen "pronoms personnels conjoints", das System der gebundenen (oder enklitischen) Personalpronomina, zeigt ein Zwei-KasusSystem, in der 3. Pers. Sing und Plur. ("Referenz" in den Worten Harald Weinrichs) sogar drei Kasus (il - le - lui; elle - la - lui; ils/elles - les - leurs) mit einer dem deutschen Dativ ähnlichen Kategorie ("partner" ${ }^{\prime 23}$ ).

\footnotetext{
${ }^{21}$ Siehe Tabellen am Ende, insbesondere schraffierte Felder.

${ }^{22}$ Von Haberland (1994) als "Non-Oblique" (oder "Subjective"), "Oblique" und "Possessive" bezeichnet.

${ }^{23}$ Cf. Weinrich (1985). Das Französische hat sich - wie die anderen romanischen Sprachen - aus dem Vulgärlatein entwickelt, einschließlich seiner Personalpronomina. Lui, zum Beispiel, hat sich aus altfranzöisch $l i$ entwickelt, das auf vulgärlateinisches $l i$ zurückgeht (im klassischen Latein illi, Dativ Sing. Mask. und Fem.). Man könnte selbstverständlich einwenden, die französischen Personalpronomina seien als Beleg für "inneren" Sprachwandel untauglich, sondern Ergebnis des Sprachkontakts zum vulgärlateinischen Superstrat. Andererseits haben alle vulgärlateinischen Formen in den Folgejahrhunderten einen morphologischen Reduktionsprozess durchlaufen. Ursprünglich hatte auch das französische Nominalsystem eine Kasusunterscheidung zwischen "cas régime" und "cas oblique" (meist Akkusativen im klassischen Latein), die jedoch auf eine einzige Form reduziert wurde. Die Kasusmorphologie im System der Personalpronomina wurde
} 
Was könnten also die Gründe des komplexeren Kasussystems im Bereich der Personalpronomina, insbesondere des Erhalts früherer oder gegenwärtiger Dativformen, sein?

Sieben Faktoren seien hier erwähnt (cf. Salmons 1994: 64f.):

1 Die hohe Frequenz der Pronomina könnte sie resistenter machen gegenüber sprachlichem Wandel.

2 Personalpronomina haben häufig belebte Referenten, die eine deutlichere morphologische Unterscheidung ihrer syntaktischen Rollen verlangen. ${ }^{24}$

3 Pronomina sind geschlossene Klassen, die offenbar auf andere Weise lexikalisch gespeichert werden als offene Klassen (siehe unten).

4 Pronomina können als Kopf einer Nominalphrase fungieren, und Köpfe scheinen eine stärkere morphologische Markierung zu tragen.

5 Personalpronomina sind häufig Suppletivformen; diese werden vermutlich individuell und als ganze Einheiten lexikalisch gespeichert (siehe unten). Der Verlust von Kasusmarkierungen an Nomina, die in "Dekomposition" gespeichert werden, ist daher weniger "einschneidend" als die Ersetzung ganzer lexikalischer Einträge.

6 Die syntaktische Reihenfolge im Mittelfeld der deutschen Verbklammer zeigt für das Personalpronomen (im Unterschied zum Nomen) die unmarkierte Anordnung: (Subjekt) - direktes Objekt (im Akkusativ) - indirektes Objekt (im Dativ). Dies korrespondiert mit der unmarkierten Reihenfolge: bekannt vor neu, unbetont vor betont. Diese Koinzidenz könnte den Dativerhalt ebenfalls unterstützen (cf. Eisenberg 1994: 383f.).

7 In der neurolinguistischen Forschung wird seit einigen Jahren diskutiert, ob Wörter im Lexikon als ganze Einheiten ("Full Listing") oder in Dekomposition repräsentiert sind. Neuere Untersuchungen scheinen darauf hinzuweisen, dass monomorphematische Wörter und polymorphematische Wörter mit irregulärer Wortbildung (ebenso wie weniger produktive morphologische Muster und semantisch weniger transparente Wörter; cf. Cholewa (1993), Tabelle am Ende) als ganze Einheiten lexikalisiert werden. Eben diese Merkmale treffen auf Personalpronomina in hohem Maße zu. Nach Kiparsky (1982) sind solche ganzheitlich repräsentierten Einträge auf der tiefsten Lexikonebene gespeichert. Auch dies könnte sprachlichen Wandel eindämmen oder verzögern.

in erheblich geringerem Maße reduziert (cf. Lausberg 1962:124ff.; Bourciez 1967: 92f., 238ff., 677ff.).

${ }^{24}$ Dies betrifft den Dativ noch stärker als den Akkusativ: Nach Jakobson gibt der Dativ häufig einen belebten Empfänger des Geschehens an: "Als Träger des D-s [Dativs, P.R.] des Adressaten fungiert meistens ein belebtes Wesen".Jakobson (1936: 274). 
Es gibt also offenbar gute Argumente anzunehmen, dass die Entwicklungsgeschwindigkeit und -reihenfolge des Kasusabbaus in den deutschen Sprachinselvarietäten in hohem Maße Ausdruck eines "typologischen" Sprachwandels ist, der nicht unmittelbar auf interlinguale oder intralinguale Konvergenz zurückgeht.

Es sei allerdings ketzerischerweise hinzugefügt, dass nicht immer klar ist, was mit "typologischem" Wandel gemeint ist: Können wir bestimmte Sprachwandelphänomene eindeutig einem wohldefinierten "Sprachtyp" zuordnen - oder ist die typologische "Verwandtschaft" nur eine Zusammenfassung für eine Anzahl von beobachteten Sprachwandelphänomenen? Möglicherweise ist mit dem Verweis auf "typologischen" Wandel noch nicht sonderlich viel erklärt. Die Reduktion der - in einer einzigen sprachlichen Form enthaltenen - komplexen grammatischen Information und die Externalisierung syntaktischer (relationaler) Merkmale könnten neurolinguistisch zu erklärende Vorgänge andeuten, die auch in anderen Fällen auftreten, etwa in Prozessen der Pidginisierung und Kreolisierung (Siehe Kap. 5 und 6).

\section{Soziolinguistik der Konvergenz}

Wenn wir oben zwischen den drei potentiellen Arten der Konvergenz unterschieden haben, der (intralingualen) Konvergenz zwischen dialektalen Varietäten, der (interlingualen) Konvergenz zwischen Sprachen und der (polyzentrischen) "Konvergenz" einer Parallelentwicklung der deutschen Varietäten unabhängig von externem Einfluss, dann haben wir dies aus Gründen der analytischen Trennschärfe getan. Wir haben eine wichtige Frage noch nicht beantwortet: Warum treten die beobachteten Entwicklungsphänomene gerade in dieser historischen Situation auf? Unter welchen Bedingungen tritt eine Beschleunigung von Konvergenz oder Sprachwandel ein? Warum sind bestimmte sprachliche Merkmale zu bestimmten Zeiten sprachlichem Wandel besonders ausgesetzt und andere nicht? In welcher soziolinguistischen Situation überdauern bestimmte sprachliche Merkmale, während andere untergehen?

Zur Erklärung werden wir die oben beschriebenen Konvergenzprozesse im Zusammenhang betrachten müssen. Dies soll an einem eindrucksvollen Beispiel geschehen:

Seit einiger Zeit zeigen auch die niederdeutsch sprechenden Russlandmennoniten einen rapiden Wandel im morphologischen System ihrer Varietäten, der im Kasusabbau ebenso wie in einem schrittweisen Verlust des Präteritums besteht (cf. Grinjowa 1990) - dies, obwohl gemeinhin der Präteritumsschwund nicht gerade als typisch für das Niederdeutsche angesehen wird; sondern eher für mittel- und oberdeutsche Varietäten.

Worauf könnte dieser rapiden Wandels zurückzuführen sein? 
Selbstverständlich sind die Mennoniten seit der erwähnten Bildung von "Zentraldörfern" (mit gemeinsamer Arbeit im Kolchos, gemeinsamem Schulunterricht der Kinder usw.) einem intensiven Varietätenkontakt mit den Sprechern anderer dialektaler Varietäten ausgesetzt. Andererseits haben die Mennoniten mehr als alle anderen Sprechergruppen - ihre Varietäten bewahren können und verwenden sie in der eigenen Gruppe, während Russisch oder die deutsche Standardsprache der Kommunikation mit anderen Deutschen dient. Das dichte mennonitische Netzwerk stellte bisher einen Rückhalt für die niederdeutschen Varietäten dar, die kaum im Kontakt mit anderen Deutschsprechenden zu verwenden sind, da nur einige ältere Nicht-Mennoniten sie verstehen, nicht jedoch die jüngere Generation (die auch kaum standardsprachliche Kenntnisse mehr hat). Dies bestätigte sich in einer Untersuchung in der Altaj-Region, Westsibirien, einem der letzten Kerngebiete russlanddeutscher Siedlungen: In der Intragruppenkommunikation der Mennoniten wird Deutsch noch zu 60\% ausschließlich verwendet, in der Intergruppenkommunikation nur zu $20 \%{ }^{25}$

Die Mennoniten haben ihr eigenes kommunikatives Netzwerk, und dies gilt sogar in gewissem Maße noch für die Jugendlichen. Dies setzt sie direktem Einfluss aus anderen deutschen Varietäten in geringerem Maße aus. Sprachlicher Wandel als Folge dialektaler Konvergenz ist daher nicht sehr wahrscheinlich.

Der schrittweise Verlust des Präteritums könnte durchaus auf russischen Einfluss zurückgehen. Die Ersetzung des Präteritums durch das Perfekt könnte durch eine intendierte "Simulation" des russischen perfektiven Verbaspekts motiviert sein. Andererseits sind die Mennoniten die Gruppe mit dem ausgeprägtesten domänenspezifischen Bilingualismus. Entlehnungen aus dem Russischen und Interferenz sind bei ihnen geringer als in anderen Gruppen. Das Russische nimmt allerdings in der Intergruppenkommunikation deutlich zu und wandert so auch in die Peer-Group und die Familie.

${ }^{25}$ Cf. Rosenberg (1994: 294). Die Studie wurde in den russlanddeutschen Dörfern Podsosnowo und Redkaja Dubrawa durchgeführt - Orte, die ich zum erstenmal mit Harald Weydt besuchte und in denen wir unsere Sprachinselforschung - unter exzessivem Wodka-Einfluss - begannen.

Die Studie umfasste etwa 750 Fragebögen, darunter die gesamte Schuljugend und jeden fünften Erwachsenen der beiden Dörfer. Zusätzlich wurde ein Soziogramm einer 11. Schulklasse erstellt, das den oben beschriebenen Effekt bestätigte: Die Schüler wurden gebeten anzugeben, wer ihre Freunde in der Klasse seien - und mit wem sie welche Sprache(n) verwendeten. Das Soziogramm zeigt deutliche Netzwerk-Cluster innerhalb der Gruppe: Multiplexe Muster verbinden vor allem Mennoniten-Mennoniten-Cluster (mit Unterschieden zwischen Jungen und Mädchen). Die Kommunikation in der Peergroup wird in Russisch und Deutsch durchgeführt. Russisch tritt in allen Clustern auf, aber hauptsächlich mit Nicht-Mennoniten. Auffällig ist der Umstand, dass Kommunikation auf Deutsch fast ausschließlich unter Mennoniten vorkommt. 
Der sprachliche Wandel unter den Mennoniten stimmt vollständig mit der oben beschrieben Entwicklungsrichtung überein, alle deutschen Varietäten zeigen ähnliche Erscheinungen. Aber die Mennoniten unterliegen ihm erst seit einiger Zeit mit erheblicher Beschleunigung. Wie könnte sich dies erklären?

Ein Wandel des Sprachsystems hängt stark von der Stabilität oder Instabilität der sprachlichen Norm ab. Normen sind Ausdruck des Bewusstseins der Distinktivität von Sprechergruppen. Normstabilität besteht vor allem in Normsicherheit und Normloyalität. Im vorliegenden Fall ist die Normsicherheit herabgesetzt durch die sprachliche (und kulturelle) Heterogenität der russlanddeutschen Mischsiedlungen. Normloyalität als einstellungsbedingte Größe zerfällt, wenn kulturelle und sprachliche Gruppengrenzen zweifelhaft werden. Eben dies ist heute auch bei den Mennoniten der Fall, die bisher weitgehend "immun" gegen äußeren Einfluss schienen: Religiöse und kulturelle Wertsysteme verblassen, Mischehen nehmen zu, räumliche und soziale Mobilität schaffen Einfallstore für den russischen Einfluss, der vor allem die jüngere Generation erfasst. Die hochdeutsche Standardsprache, die unter den Mennoniten lange Zeit als "Bibelsprache" - und angesichts ihrer Bildungsorientierung auch als Schulsprache am weitesten verbreitet war, fällt als stabilisierender Gegenpol infolge der religiösen und schulischen Restriktionen aus.

Auf ähnliche Weise scheint sich die Erosion sprachlicher Normen unter den Sprechern des Pennsylvania German in einem generationenspezifischen sprachlichen Wandel bemerkbar zu machen, wie Marion Huffines (1989) betont:

"For the nonsectarians, native speakers use Pennsylvania German among themselves and with their linguistic peers, and they switch languages as is socially appropriate. [...] Among the nonsectarians there is a sharp discontinuity in the transmission of Pennsylvania German: the present generation of native speakers chose to speak English to their children [...]. The PG norm was effectively removed from the acquisitional potential for the next generation. As Pennsylvania German dies out among the nonsectarians, one finds the loss of the PG norm reflected in faulty linguistic formulations, such as unsuccessful attempts to employ the dative case. Convergence to English is minimal. [...] Among the sectarians, Pennsylvania German continues a forced existence where sociolinguistic norms prescribe its use but not its form. Convergence toward an English model is readily apparent." (Huffines 1989: 225)

Im Hinblick auf die Normstabilität der Sprecher könnte dies folgendermaßen interpretiert werden:

Die (älteren) Native-Speaker unter den Nonsectarians weisen stabile Normen auf, bezogen auf ihre Normsicherheit, jedoch weniger auf ihre Normloyalität, da sie die Sprache nicht mehr an ihre Kinder weitergeben. Codeswitching steht hierzu nicht in Widerspruch, denn zumindest ein bewusstes Switching ist Ausdruck eines Bewusstseins sprachlicher Normen und situativer Angemessenheit. 
Die Konvergenz ist folglich bei den Älteren minimal: Der Kasusverfall im Bereich der Personalpronomina ist gering (mit einem korrekten Dativgebrauch von $80 \%$; cf. Huffines 1989: 217).

Bei den jüngeren Nonsectarians verlieren die sprachlichen Normen des Pennsylvania German an Stabilität, sowohl hinsichtlich ihrer Normsicherheit als auch besonders ihrer Normloyalität. Sie konvergieren nur in begrenztem Maße, gehen aber vermehrt zum Englischen über. Der Kasusabbau bei den Personalpronomina wächst (mit einem korrekten Dativgebrauch in der ersten primärsprachlich englisch aufgewachsenen Generation von $50 \%$, in der zweiten von $40 \%)$.

Die Sectarians zeigen eine hohe Normloyalität, aber geringe Normsicherheit. Da sie über kein distinktives Codeswitching-Verhalten verfügen, durchsetzt englischsprachiger Einfluss ihre sprachlichen Normen. Die Konvergenz ist stark, der Kasusabbau maximal (der Dativ ist nahezu aufgegeben).

Den Zusammenhang zwischen Sprachwechsel und erodierenden Gruppennormen hat auch Göz Kaufmann (1997) unter Mennoniten in Mexiko und Texas untersucht: Es zeigt sich, dass eine starke Korrelation zwischen attitudinalen und sprachlichen Daten auf eine erhebliche Heterogenität der Gruppen in ihrer Bereitschaft zum Sprachwechsel hinweist. ${ }^{26}$ Vor allem in instabilen Sprechergemeinschaften scheint der Grad der Normloyalität handlungsleitend zu sein. Kaufmann interpretiert dies mit Weinreich et al. (1968: 185f.): "in the earliest and latest stages of a change, there may be very little correlation with social factors".

Dies findet Unterstützung in neueren soziologischen Ansätzen, die Modelle für das "Framing" einer Interaktionssituation, zum Beispiel einer Sprachkontaktsituation, bereitstellen: Esser (1996a, 1996b) entwirft ein solche "Logik der Situation" aus der Sicht des sogenannten rc+ ("rational choice plus"): Die Wahrnehmung und Interpretation einer gegebenen Situation hinge vom Nutzen und der Wahrscheinlichkeit von "Frames" und "Habits" ab. Voraussetzung einer sinnvollen Situationsdeutung ist folglich, dass diese einen Nutzen und eine gewisse Wahrscheinlichkeit der Gültigkeit besitzt. Chronische und fest verwurzelte (leicht zugängliche) Einstellungen (ebenso wie "typische" und klar erkennbare

\footnotetext{
${ }^{26}$ Die jungen "progressiven" Männer unter den mexikanischen Mennoniten zeigen viele und starke Korrelationen zwischen Einstellungen und Sprachverhalten, die "Konservativen" wenige und schwache Verbindungen. Dies wird als Erklärung für den deutlichen Wandel im Sprachverhalten unter den jungen "Progressiven" herangezogen, die zunehmend von der mennonitischen "High Variety Hüagdietsch" zum Spanischen übergehen, im Gegensatz zu den "Konservativen". Die texanische Mennonitengruppe weise nur wenige und schwache Korrelationen auf, da ihr Sprachwechsel zum Englischen weitgehend vollzogen sei (cf. Kaufmann 1997: 320).
} 
Situationen) wirkten sich darin auf die Wahrscheinlichkeit aus, mit der ein tragfähiges Situationsverständnis zustandekommt. Umgekehrt schwächten eine geringe Motivation, unklare Gruppengrenzen, kognitive Dissonanzen und die $\mathrm{Au}-$ flösung kultureller oder sprachlicher Normen ein stabiles (und erst recht ein gemeinsames) Situationsverständnis ebenso wie ein automatisiertes, habituelles Verhalten (cf. Lapinski/Rosenberg 2001). Der Verfall kulturellen und sprachlichen Wissens oder das Verblassen von Gruppengrenzen kann dementsprechend eine Auflösung gemeinsam geteilter "Frames" und normativen Verhaltens einleiten. In solchen Zeiten der kulturellen Krise kann das Schwinden sprachlicher Normen den Weg freigeben für ungehinderten Sprachwandel.

Mit Blick auf die "Vitalität" von ethnosprachlichen Gruppen hat Howard Giles zunehmendes Gewicht auf die "subjective vitality" ${ }^{27}$ solcher Gruppen gelegt, die eher als Schlüssel zum Verhalten von Sprachminderheiten zu verstehen seien als das Vorhandensein "objektiver" Voraussetzungen der Gruppenvitalität (wie ein hoher Status, günstige demographische Bedingungen, institutionelle Förderung, cf. Giles et al. 1977): Für das Verhalten von Sprachminderheiten kann es wichtiger sein, welches Verständnis eine Gruppe von einer Kontaktsituation hat, als welche realen Bedingungen sie vorfindet.

Dass ein solches Verständnis nicht "autonom", sondern interaktional konstituiert wird, beschreiben Le Page and Tabouret-Keller anhand von Kreolgemeinschaften: Soziale und sprachliche Grenzziehungen seien Gegenstand symbolischer Interaktion, indem sogenannte "acts of identity" in einem Aushandlungsprozess an gruppenkonstituierendem Symbolwert gewinnen ("Fokussierung") oder verlieren ("Diffusion") (cf. Le Page/Tabouret-Keller 1985). Der genannte Verfall kulturellen und sprachlichen Wissens oder das Verblassen von Gruppengrenzen wären in diesem Sinne als Diffusionsprozesse zu verstehen.

Nach Eugenio Coseriu sind sprachliche (und kulturelle) Normen die Frontlinie sprachlichen Wandels: "Ein Sprachwandel beginnt und entwickelt sich immer als 'Verschiebung' einer Norm" (Coseriu 1974: 119). Da die Norm als die Menge dessen anzusehen ist, was in einer Sprache üblich ist, während das System das enthält, was in einer Sprache möglich ist, ist die Norm immer als das Einfallstor für den sprachlichen Wandel in den Grenzen des Systems zu betrachten (ebenso wie das System innerhalb der Grenzen des Sprachtyps). ${ }^{28}$ Systemwandel wird

\footnotetext{
${ }^{27}$ Harwood/Giles/Bourhis (1994: 175) betonen die "subjective vitality as a predictor of ethnolinguistic behaviour"; cf. auch Niedzielski/Giles (1996).

28 "Die sprachlichen Konvergenzen haben nichts Geheimnisvolles an sich [...]: es handelt sich dabei einfach um die Anwendung systematisch und typologisch analoger Muster in verschiedenen Sprachen, [...] was in der Norm als Wandel erscheint, ist vom System her
} 
daher "in Zeiten schwacher Tradition und kulturellen Verfalls oder in Gemeinschaften mit beschränkter sprachlicher Kultur besonders zahlreich sein und weitreichende Möglichkeit haben, sich auszubreiten" (Coseriu 1974: 117).

Unter den Russlandmennoniten bereitet offensichtlich der Verfall sprachlicher und kultureller Normen den Weg für einen beschleunigten systematischen und typologischen Wandel, der keineswegs durch (intra- oder interlinguale) Konvergenz verursacht ist, jedoch unter dem Druck intensiven Sprachkontakts stattfindet:

"die wichtigste Triebkraft für die Entfaltung der latenten inneren Tendenzen [besteht] gerade in der äußeren Einwirkung, in einer Reihe 'außerlinguistischer', hauptsächlich soziologischer Faktoren." (Hutterer 1987: 453, Hervorheb. im Orig.).

\section{$4 \quad$ Sprachkontakt und Sprachwandel}

Um die Frage nach intern oder extern induziertem sprachlichen Wandel genauer zu betrachten, wollen wir im folgenden Kapital auf die eingangs von Wayne O'Neil aufgeworfene Frage zurückkommen. Bezogen auf die Reduktion der Flexionsmorphologie des Altenglischen unterscheidet O'Neil zwischen "Neutralisation", die durch Sprachkontakt zustandekomme, und "Simplifikation", die durch sprachinterne Prozesse verursacht sei.

O'Neil (1978) versteht den Abbau der Flexionsmorphologie des Altenglischen als Neutralisation, da "the complex inflectional system of Old English was largely and rapidly neutralized in the northern parts of England on contact with the complex inflectional system of Old Norse" (O'Neil 1978: 249). ${ }^{29}$

Im südlichen Mittelenglisch dagegen habe eine Simplifikation stattgefunden, die nicht durch Sprachkontakt induziert gewesen sei (das Altfranzösische habe bestenfalls ihre Verbreitung begünstigt): "a continuation of the inflectional change that had been going on for centuries in the Germanic languages, this

gesehen nichts weiter als seine Anwendung; und Wandel im System ist dementsprechend Funktionieren des Sprachtyps." (Coseriu 1975: 145)

${ }^{29}$ O'Neil (1978: 262) weist darauf hin, das Altenglische und das Altnordische (Old Norse) seien nah verwandt und zeigten eine "basic underlying sameness": "Imagine [...] a situation and an area in which there are two groups of people speaking languages which because of their common origin are remarkably similar in their phonology, syntax (deep and surface), and semantics, but which for historical reasons contrast sharply in their inflections, in those markers of superficial relationships, surface structure clues on the basis of which deep structure guesses are made. In such a situation, granting that the two groups are reasonably at peace and willing to mix and trade back and forth, how could communication be improved - indeed, made possible? Easy: radically and quickly simplify the inflectional noise." (O'Neil 1978: 257) 
change itself a result of a balancing off of the learnability of inflections against their perceptibility" (O'Neil 1978: 256).

O'Neil vergleicht diese Entwicklungen mit anderen Vorgängen der Neutralisation und Simplifikation ${ }^{30}$ und folgert daraus als generalisierte Hypothese:

"If there is significant and more-or-less permanent contact between two closely related languages differing for the most part only in superficial aspects of their grammars (inflections, accent, tone, etc.) these superficial differences will be rapidly neutralized or erased. [...] Without language contact, inflectional systems will simplify only so far as there is room for easing learning without greatly decreasing perceptibility." (O'Neil 1978: 283)

Neutralisation wird als soziolinguistisches Phänomen betrachtet, als "sudden response (in a generation or two) to a pressing problem of communication", the problem "to ease understanding" (O'Neil 1978: 275). Simplifikation sei demgegenüber ein psycholinguistisches Phänomen: "a function of a languageinternal move to ease learning, to rebalance an imbalanced learnability-perceptibilty state" (O'Neil 1978: 282). Neutralisation geschieht durch Erwachsene, "forming a new language community", Simplifikation durch Kinder, "learning the language of their community" (O'Neil 1978: 283).

O'Neil's Hypothesen zeigen eine gewisse Attraktivität, vor allem weil sie eine Bresche schlagen in die Barriere, die häufig zwischen grammatischem Wandel (des Flexionssystems) und Sprachkontaktphänomenen aufgerichtet wird.

Natürlich ist die Annahme eines auf Sprachkontakt zurückgehenden Abbaus des altenglischen morphologischen Systems nicht neu, wenn auch meist der Einfluss des Französischen herangezogen wird, dessen Nominalflexion bereits partiell reduziert war. Der ausschlaggebende Punkt in der Argumentation O'Neils ist jedoch der Verfall des morphologisch reichen Flexionssystems des Altenglischen im Kontakt mit dem morphologisch reichen Flexionssystem des Altnordischen.

Die postulierte strikte Trennung zwischen Neutralisation und Simplifikation verursacht allerdings einige neue Probleme und lässt andere Probleme ungelöst:

Sprachkontakt mag die Vorgänge des Sprachwandels im Altenglischen beschleunigt haben, sie waren jedoch mit hoher Wahrscheinlichkeit bereits im Gange,

\footnotetext{
${ }^{30}$ Prozesse der Neutralisation sieht er im Zusammenbruch des Flexionssystems im Afrikaans, der auf den Kontakt zu niederländischen Mischdialekten und zum Niederdeutschen in Kapstadt zurückgeht, sowie im Abbau der Flexionsmorphologie in den kontinentalskandinavischen Sprachen aufgrund des Kontakts zum Mittelniederdeutschen. Simplifikation finde im Isländischen aufgrund seiner Isolation statt und im Hochdeutschen wegen seiner generellen Entfernung vom Germanischen (cf. O'Neil 1978: 275ff.).
} 
wie etwa die Reduktion der Verbflexion,z. B. der Einheitsplural (für die erste, zweite und dritte Person) im Präsens und im Präteritum, in der starken und schwachen Verbkonjugation.

Ferner waren auch die skandinavischen Sprachen (außer dem Isländischen und Färöischen) einer radikalen Erosion des Flexionssystems ausgesetzt. O'Neil (1978: 267) begründet diese mit dem intensiven Sprachkontakt zum Mittelniederdeutschen. Diese Kontakte waren jedoch sicherlich von deutlich anderer Natur als jene zwischen den Sprechern des Altenglischen und des Altnordischen. Ein vergleichbar intensiver Sprachkontakt mit dem Mittelniederdeutschen war auf die Handelszentren der Hanse beschränkt, es expandierte flächendeckend nur in Dänemark. ${ }^{31}$

Die Annahme, dass die Welt des Sprachwandels in zwei Teile zerfällt - Neutralisation mit Sprachkontakt, Simplifikation ohne - scheint doch zu einfach zu sein. Dies gesteht O'Neil selbst ein, wenn er auf "middle state"-Fälle wie das Färöische hinweist. ${ }^{32}$ Seine Erklärung ist, dass das Färöische in doppeltem Kontakt gestanden habe: "subject to contact with closely related languages of two extreme inflectional persuasions" (O'Neil 1978: 280). Die kommunikativen Erfordernisse des Kontakts zu den Sprechern beider Sprachen hätten den Wandel in der einen wie der anderen Richtung begrenzt. Dies allerdings ist in gewissem

${ }^{31}$ Es sei nicht verkannt, dass das Dänische im Mittelalter die dominante Sprache unter den skandinavischen Sprachen war, und es könnte eine vermittelnde Rolle in der Verbreitung (kontaktbedingter) Sprachwandelphänomene gespielt haben. Auch ist es zutreffend, dass das Norwegische über lange Zeit durch das Dänische geprägt wurde, wie auch das Schwedische durch das Norwegische und Dänische. Jedoch waren im Altdänischen Sprachwandelvorgänge bereits im Gange, als die Kontakte zum Mittelniederdeutschen zunahmen, lange bevor etwa die Lübeckische Schriftsprache zur wichtigsten Sprache des Ostseeraums wurde.

Auf die frühe altdänische Zeit bezogen äußert Hutterer (1987: 174) explizit: "Die Zersetzung der Nominalflexion war schon im Gange ". Er erwähnt den Zusammenfall von Nominativ und Akkusativ. (Das Altschwedische entwickelte sich in gleicher Richtung zu eben dieser Zeit.) In den Seeland-Dialekten blieb lediglich eine Unterscheidung zwischen Einheitskasus und Genitiv übrig, im Dialekt von Jütland, unmittelbar an das niederdeutsche Sprachgebiet angrenzend, wurde die Nominalflexion fast vollständig beseitigt. Eben dies ist mit dem obigen Einwand gemeint, Sprachkontakt habe Vorgänge der Simplifikation eher beschleunigt als verursacht.

${ }^{32}$ Das Färöische, die Sprache der Inseln auf halbem Wege zwischen Skandinavien und Island, wird von O'Neil als "middle state"-Flexionssystem "between the complexity of Icelandic and the simplicity of the continental Scandinavian languages" (O'Neil 1978: 277) angesehen. O'Neil (1978: 280) verweist jedoch selbst auf das damit verbundene logische Problem: "although it is interesting to contemplate the hypothetical state of half-isolation, half-contact, it finally makes little sense: there is either isolation or contact, and then their attendant consequences - simplification or neutralization." 
Sinne unbefriedigend, da das Färöische morphologisch dem Isländischen bei weitem näher steht als den Skandinavischen Sprachen, soweit dies das Flexionssystem betrifft ${ }^{33}$ : Seine Nominalflexion ist isländisch ohne den Genitiv (der jedoch bei Eigennamen existiert), seine Verbflexion ist isländisch mit Einheitsplural. ${ }^{34}$

Das ungelöste Hauptproblem bleibt jedoch nach O'Neil die Erklärung der Simplifikation: "what triggers off simplification is not at all clear" (O'Neil 1978: 284). Er sieht - wie oben erwähnt - als ihr Motiv eine sprachinterne Entwicklung, die "Lernbarkeit" ("learning burden") zu erleichtern (ohne die Wahrnehmbarkeit "tiefenstruktureller" Unterscheidungen zu tangieren ${ }^{35}$ ).

Aber werden die Sprachen der Welt tatsächlich immer einfacher? Folgen sie einer Art von Lernökonomie? Das Wesen der Sprache, von endlichen Mitteln unendlichen Gebrauch zu machen, ist sicherlich ein hochökonomisches Prinzip. Aber es entstehen ständig neue Unterscheidungen: Sprachen, die morphologisch einfacher werden, ersetzen die verloren gegangenen grammatischen Distinktionsmittel durch andere Ausdrucksmittel wie neue Lexikoneinträge oder Wortstellungsrestriktionen. Artikel mit durchaus komplizierten Strukturen (bestimmter, unbestimmter, Null-Artikel) entwickeln sich, Präpositionen enkodieren komplexe semantische Konzepte ${ }^{36}$, Hilfsverben entstehen und verschmelzen miteinander zu einem irregulären Subsystem von Suppletivformen und Stammallomorphie.

Die Vorstellung einer sprachinternen, von Sprachkontakt gänzlich unbeeinflussten Simplifikation könnte sich als "Papierkorb-Kategorie" erweisen, in der alle Vorgänge untergebracht werden, für die eine plausible Erklärung noch aussteht.

Warum (und wann) empfinden Lerner einer Sprache es als notwendig, die "Last des Lernens" zu erleichtern?

\footnotetext{
${ }^{33}$ Sein phonologisches System ist eng verwandt mit den südnorwegischen Dialekten; cf. Hutterer (1987: 160).

${ }^{34}$ Natürlich sind die Färöer seit dem Mittelalter durch Norweger und Dänen kolonisiert worden (sie waren Teil des Norwegischen Königreichs, dann - mit Norwegen - Teil Dänemarks), aber das war auch das Schicksal Islands. Möglicherweise ist das Fehlen einer Schriftsprache und der vorwiegende Gebrauch mehrerer, sehr unterschiedlicher dialektaler Varietäten eine plausiblere Erklärung für die Simplifikationen im Flexionssystem des Färöischen.

${ }^{35}$ Numerus und Tempus werden als tiefenstrukturelle Unterscheidungen angesehen (cf. O'Neil 1978: 261).

${ }^{36}$ Figur-Grund- oder "Trajector-Landmark"-Konzepte in der Begrifflichkeit Langackers (1999), die anderen (syntaktischen oder Kompositions-) Strukturen ebenfalls zugrundeliegen.
} 
Und warum tritt von Zeit zu Zeit ein beschleunigter Veränderungsprozess ein, der doch nichts grundsätzlich anderes erzeugt als der Sprachwandel in "SlowMotion"?

Ist der Zusammenfall der Konjugationsformen für die 2. und 3. Pers. Sing. (Ind. Präs.) im Isländischen (sit - situr - situr) fundamental verschieden von dem Zusammenfall aller drei Personen im Norwegischen (sitter - sitter - sitter), oder sind dies lediglich verschiedene Entwicklungsstufen?

Sind - umgekehrt - die Grenzen der Neutralisation und der Simplifikation von völlig unterschiedlicher Art? Simplifikation fände nach O'Neil ihre Grenze in der Unterscheidbarkeit tiefenstruktureller Kategorien. Die Entlehnung des Personalpronomens der 3. Pers. Plur._eim aus dem Altnordischen, die im nördlichen Mittelenglisch zu aim (neuengl. them) führte, ersetzte die altenglische Dativform him für die 3. Pers. Plur., die zusammengefallen war mit der 3. Pers. Sing. him - und sorgte so für die Unterscheidbarkeit von Singular und Plural. Offensichtlich fand auch die "kontaktbedingte" Neutralisation ihre Grenze in der Aufrechterhaltung "tiefenstruktureller" Distinktionen (wie des Numerus).

Neutralisation könnte sich als Sonderfall der Simplifikation erweisen, als Hochgeschwindigkeits-Simplifikation, die jedoch nicht grundsätzlich verschieden von jener ist, keine anderen Elemente erfasst, keine unterschiedliche Entwicklungsrichtung nimmt und im Zusammenspiel interner und externer Triebkräfte ebensowenig monokausale Ursachen besitzt.

Wie wir am Beispiel der Russlanddeutschen haben feststellen können, scheinen Sprachkontakt und sprachlicher Wandel (wie etwa morphologische Simplifikation) ineinanderzugreifen. In Zeiten ernsthafter Norminstabilität kann es zu "galoppierendem" Wandel kommen: Die Normloyalität geht zurück, wenn kulturelle und sprachliche Gruppengrenzen undeutlich werden. Die Normsicherheit kann rapide zerfallen unter intensivem Sprachkontakt, der die Herausbildung von Mischvarietäten oder sogar Sprachmischung bewirkt - bis zu dem Punkt, an dem die Grenze zwischen Ingroup und Outgroup verblasst oder schwindet.

Dies aber ist nicht weit entfernt von den Merkmalen, die uns aus Untersuchungen über Pidgin- und Kreolsprachen bekannt sind. Es könnte daher sinnvoll sein, Ähnlichkeiten zwischen Sprachinseln in Auflösung und Pidgin- und Kreolsprachen eingehender zu betrachten.

\section{$5 \quad$ Konvergenz, Sprachwandel und Pidginisierung}

Der Abbau des morphologischen Systems, insbesondere der Kasussynkretismus, gehört zu den Hauptkennzeichen fast aller deutschen Sprachinselvarietäten, unabhängig davon, ob sie dem Kontakt mit kasusmarkierenden oder nicht kasusmarkierenden Sprachen ausgesetzt sind. 
Die starke Reduktion der Flexionsmorphologie der Sprachinselvarietäten ist offenbar kein Resultat von Interferenzen aus den Kontaktsprachen, sondern in vielen Fällen des Abbaus der grammatischen Systeme:

"Case syncretism or case merger [...] characterizes terminal stages of receding languages." (Huffines 1989: 212)

Tatsächlich kann man diese Abbauprozesse als Stufen eines Vorgangs ansehen, der gelegentlich als "language contraction", "language obsolescence" oder auch als "Sprachtod" bezeichnet worden ist. Sprachinseln in "Auflösung" weisen einige Parallelen mit Pidginisierungsprozessen auf, die sowohl sprachstrukturelle Ähnlichkeiten als auch soziolinguistische Gebrauchsbeschränkungen betreffen:

"Pidgins can be recognized as a special or limiting case of reduction in form resulting from restriction in use." (Romaine 1989: 370) ${ }^{37}$

Dressler/Wodak-Leodolter (1977) gehörten zu den ersten, die auf analoge Prozesse zwischen "Sprachtod" und Pidginisierung hinwiesen. ${ }^{38}$ Peter Trudgill (1978) bezeichnet den "Sprachtod" als "creolization in reverse".

Was ist der Gegenstand dieser Analogie?

Suzanne Romaine (1989: 379) nennt vorrangig den Verlust produktiver morphologischer Muster und weist hierzu besonders auf den Kasussynkretismus und die Tendenz zur Beseitigung von Allomorphie hin. ${ }^{39}$ Pidgins haben typischerweise eingeschränkte Wortbildungsregeln (aber erwerben diese bei Expansion).

\footnotetext{
${ }^{37}$ Suzanne Romaine (1989) vergleicht "the similarities and differences between dying languages, pidgins, creoles, and immigrant languages". Der Vergleich bezieht sich auf den sociolinguistischen Kontext und auf ähnliche sprachliche Entwicklungen: Vergleichbare Sprachkontaktbedingungen sieht begründet in "basic inequalities which may exist between the users of languages with differing prestiges, utility, and recognized legitimacy in situations of contact [...]: encroaching diglossia, failure of children to acquire the mother tongue, schooling in a second language, resettlement, dispersion and intermarriage." (Romaine 1989: 371) Ähnliche sprachliche Entwicklungen bestehen in: "convergence, loss of morphological and syntactic complexity, and an overall increase in semantic transparency." (Romaine 1989: 375)

${ }^{38}$ Es war das Verdienst von Nancy Dorian, ein Forschungsfeld konstituiert zu haben, das sie als "language obsolescence" bezeichnet. Gegenstand sind die partiell gemeinsamen Vorgänge, die bis dahin unter Termini wie "language contraction", "death", "attrition", "shrinkage" behandelt wurden (cf. Dorian 1989) - Begriffe, die einer mehr oder weniger geglückten Wissenschaftsmetaphorik entstammen.

39 Allomorphie würde ersetzt durch feste und invariable Wortstellungsregeln: Das Rabaul Creole German beispielsweise habe ausschließlich SVO-Wortstellung sowohl in Matrix- als auch in subordinierten Sätzen. (cf. Romaine 1989: 376)
} 
"A preference for analytical, as opposed to synthetic, syntax is a hallmark of both pidgins and dying languages". (Romaine 1989: 379)

Auch wenn die aufgeführten Parallelen augenfällig sind, bleibt dennoch zu fragen, ob sich die Analogie lediglich auf Oberflächenphänomene einer morphologischen "Simplifikation" bezieht, die jedoch unter Umständen auf vollständig unterschiedlichen Vorgängen beruhen. ${ }^{40}$

Immerhin scheinen auch bei den Sprechern eine Reihe von analogen Prozessen zugrundezuliegen, die beachtenswert sind:

Campbell/Muntzel (1989: 185) sehen Parallelen in dem Kontinuum der sprachlichen Fähigkeiten ("proficiency continuum"), die bei Sprechern subordinierter Sprachen einem Kreolkontinuum ähneln könnten (cf. ebenso Romaine 1989: 371). Dieses Kontinuum sei hauptsächlich durch das Alter und die Spracheinstellungen der Sprecher determiniert.

Bei Sprachen mit drastisch zurückgehendem Gebrauch oder starken Funktionseinschränkungen sei es häufig schwer zu entscheiden, "who is a speaker of the language or a member of the community which speaks a particular language" (Romaine 1989: 371) Dies kennzeichnet die Lage in einer Reihe deutscher Sprachinseln sehr präzise - und es ist nicht allein ein Problem des Sprachforschers, sondern der Sprecher selbst, das die sprachliche Grenzmarkierung der Sprechergemeinschaft betrifft. Es markiert den Übergang von Sprachminderheiten zu ethno-kulturellen Minderheiten, bei denen die Sprache selbst keine Rolle mehr spielt.

Die Jugend stellt generell eine innovative Kraft dar, die im Generationenwechsel für sprachlichen Wandel sorgt. In sich auflösenden Sprachgemeinschaften wird ihr eine besondere Rolle zugemessen, da sie in Zeiten schwindenden Normbewusstseins wenig korrektiven Druck durch die Erwachsenen erfahren. ${ }^{41}$

\footnotetext{
${ }^{40}$ Mufwene (1998: 316ff.) wirft die Frage auf, ob Sprachkontakt nicht in jedem Fall von Sprachwandel eine ausschlaggebende Rolle spielt, nicht allein bei Kreolsprachen: "If the origins of creoles are so special, we would indeed like to know how special they are. [...] To be sure, there are differences between the ecologies in which creoles developed and those in which languages putatively changed normally. [...] I contend that the ecological factors and selective restructuring which produced creoles are of the same kind as those which produced 'normal' language change. Contact, I argue, is a critical factor in almost any case of language change. [...] Creolization is a social, not a structural, process".

${ }^{41}$ Romaine vermutet, dass in instabilen Sprachgemeinschaften die Jugend als Innovationskraft sehr viel weiterreichende Spielräume habe als in "normalen" Gemeinschaften: "It may be that in a community of expanded-pidgin or dying-language speakers the innovations of children have a better chance of catching on than in 'normal' communities. [...] In normal communities the expectation is that adults act as brakes in the innovations produced by children [...]. In the case of dying and pidgin languages it may be that the
} 
Sprachlicher Wandel beginnt stets mit individuellen Neuerungen (die selten durch Linguisten rekonstruierbar sind). Die wichtigere Frage ist jedoch, wie und warum sich solche Neuerungen über die Sprachgemeinschaft verbreiten können (cf. Coseriu 1974: 127).

Geringe Normativität ist offenbar das Einfallstor solcher Innovationen sowohl in sich auflösenden Sprachgemeinschaften als auch in Pidgingemeinschaften: "Some of the similarities which exist between dying languages and creoles may be due [...] to the relaxing of the norms." (Romaine 382)

Bei geringem Normbewusstsein in einer sich auflösenden Sprachgemeinschaft kann das Bedürfnis, "to ease the learning burden", zu drastischem Abbau komplexer Strukturen führen.

Campbell/Muntzel (1989: 189) sehen die sukzessive Auflösung sprachlicher Normen darin, dass obligatorische Regeln nach und nach optional werden. Was sie im einzelnen damit beschreiben, trifft die Art des sprachlichen Wandels in den deutschen Sprachinseln exakt: Sie beobachten eine "Übergeneralisierung unmarkierter Merkmale", d.h. eine "Tendenz, markierte Formen durch weniger markierte zu ersetzen [...], wenn Unterscheidungen verloren gehen, ist es das markierte Element einer Opposition, das verloren geht" (Campbell/Muntzel 1989: 187, Übers. P.R.). Romaine argumentiert in ähnlicher Richtung: "contact involves a progressive unmarking" (Romaine 1989: 382).

Selbstverständlich kann sprachlicher Wandel häufig als durch Sprachkontakt ausgelöst angesehen werden. Aber die "Anfälligkeit" für Sprachwandel und die Richtung eines solchen Wandels seien nach Campbell/Muntzel bestimmt durch sprachstrukturelle Faktoren: "the nature (marked or unmarked) of the linguistic phenomena in the structure of the dying language which leads to loss (i.e. 'internal factors')" (Campbell/Muntzel 1989: 188).

children have greater scope to act as norm-makers due to the fact that a great deal of variability exists among the adult community. [...] Simplified forms can persist in the speech of children [...] because there is little corrective pressure or model."(Romaine 1989: 372f.)

Dies mag übrigens auch ein Grund sein, warum von zahlreichen Sprachkontaktforschern die Domäne der Familie als entscheidende Instanz für Sprachbewahrung oder Sprachwechsel gesehen wird (cf.u. a. Haugen 1989).

${ }^{42}$ Ein Abbau markierter Elemente zugunsten unmarkierter liegt bereits in der Logik des Jakobsonschen Ansatzes, der zunehmende Markiertheit als zunehmende Beschränkung versteht: "Je mehr Korrelationsmerkmale der Kasus in sich trägt, desto vielfältiger wird die Geltung des bezeichneten Gegenstandes in der Aussage beschränkt und herabgedrückt" (Jakobson 1936: 282). 
Aus einer anderen Perspektive, nämlich der der Zweitspracherwerbsforschung, betrachtet Andersen (1989) "language contraction". ${ }^{43}$ Er kommt zu einem ähnlichen Ergebnis: "'simplification' is the surface result of a process whereby each linguistic form is uniquely linked to one and only one intended meaning (or function) and that language transfer (or interference) operates only in conjunction with this and related cognitive principles" (Andersen 1989: 386). ${ }^{44}$

Das sogenannte "One-to-One Principle" bei Andersen und der Verweis auf Markiertheitsstrukturen bei Campbell/Muntzel beschreiben den gleichen Sachverhalt: Andersen (1982: 95) geht davon aus, dass im Rahmen von Simplifizierungen das Merkmal verwendet wird, das eine größere "funktionale Belastung" besitzt, d.h. in einer größeren Zahl von Anwendungsfällen "passt". Dies stimmt mit dem "unmarkierten" Element bei Campbell/Muntzel (1989) weitgehend überein. Auf die Kasusreduktion in den deutschen Sprachinselvarietäten bezogen, scheint dies dem Akkusativ zu entsprechen. ${ }^{45}$

In instabilen Sprachgemeinschaften mit herabgesetzter Normativität besteht offenbar ein günstiges Terrain für eine Vereinfachung der sprachlichen Strukturen, für das, was als "typologischer" Wandel bezeichnet werden kann. Wenn wir diese Vereinfachungsprozesse genauer erklären wollen, müssen wir uns der Frage stellen, welche Strukturen vereinfacht werden und welche nicht: Wie wir

43 "Language contraction is typically a phenomenon of second language development in that the weaker, contracting language is almost always a secondary language for the speaker, even though it may be the speaker's original mother tongue" (Andersen 1989: 386).

${ }^{44}$ Diese beiden Prinzipien sind das sogenannte "One-to-One Principle" und das "Transfer to Somewhere Principle" (Andersen 1989: 386). Beispiele für das "One-to-One Principle" im Zweitspracherwerb seien: SVO-Wortstellung im Deutschen (auch in subordinierten Sätzen), postverbale Position des Objets im Französischen und Spanischen (auch von Pronomina), Stellung des Negators direkt vor die zu negierende Einheit im Deutschen und Englischen, Reduktion von (redundanten) Kongruenzmarkern.

Das "Transfer to Somewhere Principle" bedeutet, dass Transfer dann und nur dann auftritt, wenn das "One-to-One Principle" (und andere) mit der L1-Struktur übereinstimmen oder wenn die L2 ein Modell zur (Über-) Generalisierung bereitstellt. Im Transfer werden freie, invariante, funktional einfache und häufige Formen bevorzugt, die mit L1 und L2 kongruent sind. Ein Beispiel für dieses Prinzip ist die postverbale Stellung des Nominalobjekts im Französischen, das englischsprachigen Franözischlernern als Modell dient, auch Pronomina hinter das Verb zu stellen. Französischsprachige Lerner des Englischen finden kein Modell für die präverbale Stellung des Objekts im Englischen vor und verwenden daher keine französischen Regeln in ihrer "Interlanguage" des Englischen (cf. Andersen 1989: 388ff.).

${ }^{45}$ Andersen (1989: 392) hält die Kasusreduktion bei Sprechern des Pennsylvania German, die - wie oben beschrieben - die Norm der Nonsectarians aufgeben (und den Dativ durch den Akkusativ ersetzen), für eine eindeutige Anwendung des "One-to-One Principle". 
oben für die Personalpronomina gezeigt haben, ist der morphologische Abbau rascher und intensiver bei weniger "einschneidenden" Veränderungen. Solche nicht ganzheitlich lexikalisch gespeicherten - Elemente sind offenbar Gegenstand der (Über-) Generalisierung grammatischer Regeln und damit der Vereinfachung der Flexionsstruktur.

Wir stehen also immer noch vor der Frage, wie Prozesse der "Simplifikation" zu erklären sind. Noch einmal: Was ist "Simplifikation"? Und welche sprachlichen Strukturen sind in besonderer Weise "anfällig" für Vereinfachungen? Um einer Beantwortung dieser Fragen näher zu kommen, seien im folgenden Kapitel einige Überlegungen zur kognitiven Repräsentation der betroffenen sprachlichen Strukturen angeschlossen.

\section{Sprachliche Vereinfachung und kognitive Strukturen}

Steven Pinker (2000: 256ff.) betrachtet Simplifikationen in vielen Fällen als Transformationen in sogenannte "Defaultregeln": Die Ausbreitung des schwachen Verbkonjugationsparadigmas (d.h. die Markierung des deutschen Präteritums durch -te, des englischen durch -ed anstelle der starken Konjugation durch Stammallomorphie) oder des Pluralmarkers $-s$ seien solche "Defaultregeln". Pinker zufolge seien irreguläre (unregelmäßige) Formen lexikalisch repräsentiert. Eine "Defaultregel" sei "ein Flexionsmuster, das sie auf beliebige Wörter einer Kategorie anwenden können, auch wenn das betreffende Wort nie mit diesem oder irgendeinem anderen Muster im Gedächtnis gespeichert worden ist" (Pinker 2000: 253). Defaultregel sind also Regeln, die über der bloßen Kategorie (eines "Nomens" oder eines "Verbs" zum Beispiel) arbeiten. ${ }^{46}$

Wenn wir nun, wie oben beschrieben, in deutschen Sprachinselvarietäten einen fast vollständigen Flexionsabbau bei Nomina, Artikeln sowie Possessiv- und Demonstrativpronomina beobachten, nicht jedoch in gleicher Weise bei den Personalpronomina, könnte dies auf unterschiedliche kognitive Repräsentationen dieser Formen hindeuten?

\footnotetext{
${ }^{46}$ Der Präteritumsmarker - $t$ (englisch $-e d$ ) werden ebenso wie der Pluralmarker - $s$ (in beiden Sprachen) als "defaults" betrachtet, verwendbar "in all den ,Notfällen', in denen Gedächtnis und Analogie die Segel streichen - bei ungewöhnlichen Wurzeln, nicht assimilierbaren Lehnwörtern, Namen, Akronymen, Kurzwörtern, Phrasen und Zitaten. [...] Die Regularität beruht [...] auf der Fähigkeit des menschlichen Geistes, symbolische Regeln zu erwerben - Operationen, die vorbehaltlos auf jedes Exemplar einer Kategorie anwendbar sind" (Pinker 2000: 269). Pinker zufolge enthält die Sprache "Wörter und Regeln": Wörter gehörten zu einem assoziativen "Gewusst was"-System, Regeln zu einem auf symbolischen Kombinationen beruhenden "Gewusst wie"-System (Pinker 2000: $342)$.
} 
Personalpronomina, insbesondere der 3. Pers. Sing. und Plur., sind hoch suppletive Formen, wahrscheinlich individuell lexikalisiert und als ganze Einheiten auf der "tiefsten" Lexikonebene repräsentiert. Aber was bedeutet in diesem Zusammenhang die Metapher der "tiefsten" Lexikonebene?

Wir könnten mit Roman Jakobsons "Regressionshypothese" argumentieren, die davon ausgeht, dass Sprachabbau Spracherwerb in umgekehrter Richtung ist: Sprachliche Strukturen, die im Spracherwerb zuerst erscheinen, werden im Sprachabbau bis zuletzt bewahrt, Strukturen, die zuletzt erworben werden, gehen zuerst verloren (cf. Jakobson 1969).

Diese Erwerbssequenz kann bis zu einem gewissen Grade die spezifische Art des Kasusabbaus erklären, soweit dies die Unterschiede zwischen Nominalflexion und der der Personalpronomina betrifft: Der Akkusativ wird gewöhnlich vor dem Dativ erworben, Präpositionen mit Akkusativ vor solchen mit Dativ. Die Unterscheidung zwischen "statischem" Dativ (wo?) und "direktionalem" Akkusativ (wohin?) wird spät gelernt. Dativformen der Personal- und Demonstrativpronomina sind aber die ersten, die von Kindern erworben werden.

Von den Demonstrativpronomina abgesehen, stimmt diese Reihenfolge vollständig mit unseren Beobachtungen zum Kasusabbau in den deutschen Sprachinseln überein: Alle früh erworbenen Formen erhalten sich am längsten in den Sprachinselvarietäten.

Andererseits ist auch der Akkusativ mitunter Gegenstand des Kasusabbaus (bei Tilgung jeglicher Flexionsmorpheme und folglich Zusammenfall mit dem Nominativ), und auch das Demonstrativpronomen verliert den Dativ. Anscheinend sind kasusmarkierende Flexionsmorpheme "anfälliger" für eine Reduktion als ganzheitlich lexikalisierte ("full listed") Pronomina. Offenbar sind Nomina sowie Possessiv- und Demonstrativpronomina, die in Dekomposition gespeichert werden, Vereinfachungen ("Simplifikation") eher ausgesetzt.

Dies könnte als Erklärung dienen für einen Flexionsabbau bei Nomina, Possessiv- und Demonstrativpronomina sowie Artikeln, der schrittweise den Dativmarker -em in -en transformiert (mit dem Akkusativ zusammenfallend), diese Form zu ("langem") - $n$ reduziert, das schließlich optional wird oder getilgt wird. Ein solcher stufenweiser Abbau lässt sich in zahlreichen dialektalen Varietäten des Deutschen (einschließlich der Sprachinselvarietäten) beobachten. ${ }^{47}$ Da Personalpronomina wahrscheinlich nicht in dekomponierter Form (als Wurzelmorphem plus Derivations- oder Flexionsmorphem) lexikalisch gespeichert sind,

${ }^{47}$ Rosenberg (1986: 348-375) enthält etwa 1.000 Belege dieser Art aus schriftlichen Arbeiten von Berliner Schülern. O'Neil (1978: 254ff.) beschreibt den gleichen Prozess als "Simplifikation" der Nominalfllexion im Mittelenglischen Südenglands. 
sind sie vermutlich nicht in gleicher Weise zugänglich für Reduktionen der Kasusmarkierungen sowie für Übergeneralisierungen grammatischer Defaultregeln.

Selbstverständlich schützt eine "tiefe" lexikalische Repräsentation nicht vor jeglichem Sprachwandel. ${ }^{48}$ Jedoch scheint eine Transformation in Defaultregeln den Prozesss der Vereinfachung und der Regelübergeneralisierungen zu erleichtern.

In ähnlichem Sinne interpretiert Caroline Smits (1996: 47ff.) die von ihr festgestellten Sprachwandelerscheinungen im Iowa Dutch, das intensivem Sprachkontakt ausgesetzt ist. ${ }^{49}$ :

Sie unterscheidet die drei oben genannten Arten des Wandels: Regularisierung, Simplifizierung und Verlust der Flexion (bei Ersatz durch andere Mittel). ${ }^{50}$ In diesem und anderen Fällen liegt eine Interpretation der drei Arten des Sprachwandels als Stufen in chronologischer Reihenfolge auf der Hand.

Wenn die "Regularisierung" häufig den Auftakt bildet für weitergehende Vereinfachungen der morphologischen Struktur, fragt sich, was die Überführung unregelmäßiger in regelmäßige Bildungen begünstigt. Pinker (2000: 363) nennt einige Bedingungen für eine solche Regularisierung im Sinne der Transformation in Defaultregeln: Regelmäßige Formen werden gebildet (oder unregelmäßige in regelmäßige verwandelt), wenn ein Wort neu, selten oder

${ }^{48}$ Natürlich sind auch Personalpronomina dem Kasusabbau ausgesetzt, wie etwa der Ersetzung von $-m$ durch $-n$. Dies könnte sich allerdings auch - wenn es nicht Ausdruck eines phonologischen Prozesses ist - als Anwendung einer Defaultregel erweisen, nach der $-n$ als universeller Marker für den Einheitskasus oder den Obliquenkasus dient. Bezeichnenderweise ist die Ausbreitung des Akkusativs bei ihm/ihn stärker als bei mir/mich.

${ }^{49}$ Smits berichtet von einem dramatischen Sprachwandel, der mit massivem Sprachkontakt und einem Schwinden sprachlicher Normen einhergeht: "Iowa Dutch is not developing into a newly crystallized language. [...] Instead, the language is disintegrating, meaning that the grammatical system is collapsing - slowly but steadily - while language norms are disappearing." (Smits 1996: 58). In Smits (1998: 383) charakterisiert sie Iowa Dutch mit van Coetsem (1995) als Fall von "imposition", der sich in tiefgreifendem grammatischen und phonologischen Sprachwandel und in "imperfect proficiency" zeige.

50 "a.) The extended use of the rule system of the recipient language (regularization). This means that the rule system remains intact, but that the domain in which it is applied is extended at the expense of irregular forms. [...] b.) The original inflectional system of the recipient language is changed in such a way that it becomes simpler (simplification), while meaning distinctions are preserved. [...] c.) The inflectional devices for the expression of a distinction have been lost - or are avoided - because speakers are insecure about their application. This is compensated for by means of the use of other devices offered by the recipient language itself, often in the form of periphrastic constructions" (Smits 1996: 47f.). 
ungewöhnlich ist, wenn es keine normale Wurzel besitzt oder deren Information nicht auf das gesamte Wort übertragen werden kann. ${ }^{51}$

Wie wir erkennen, liegen die als günstig für die Bildung regelmäßiger Formen erwähnten Bedingungen nicht weitab von den oben beschriebenen Instabilitätsfaktoren aufgrund intensiven Sprachkontakts: Auftreten neuer oder unbekannter Wörter, Entlehnungen, Verblassen sprachlichen Wissens, Unsicherheit über sprachliche Normen.

Die historische Verbreitung der Präteritummarkers -te bzw. -ed und des Pluralmarker $-s$ wird von Pinker (2000: 270) selbst in Zusammenhang mit dem destabilisierenden Einfluss des Französischen gebracht und der Neigung, die "Notfallendungen" an alle neuen (französischen) Wörter zu setzen. ${ }^{52}$

Offenbar haben wir es also mit zwei Prozessen des grammatischen Wandels zu tun: Unregelmäßige Formen werden - mittels Defaults - in regelmäßige Formen transformiert ("Regularisierung"), und regelmäßige Formen werden vereinfacht ("Simplifizierung"), etwa in Gestalt des Kasussynkretismus, gelegentlich bis zum Stadium des vollständigen Verlusts von Distinktionskategorien.

In Zeiten der kulturellen "Krise" kann die Auflösung der Sprachgemeinschaft die Normstabilität, d.h. die Normsicherheit und die Normloyalität, drastisch verringern. Wenn die Normsicherheit (und die Normloyalität) abnehmen, wird sprachliches Wissen nur noch unzureichend weitergegeben. In diesem Fall geraten sprachliche Strukturen in Vergessenheit, Lerner empfinden den Spra-

${ }^{51}$ Im einzelnen werden nach Pinker (2000: 274f.) regelmäßige Formen gebildet (oder unregelmäßige Formen in regelmäßige verwandelt), wenn:

- ein neues Wort nicht wie ein bekanntes (unregelmäßiges) klingt (z.B. abgezockt, snarfed)

- eine unregelmäßige Form selten gebraucht wird (z.B. pflegen - gepflogen, chide chid)

- ein Wort ohne eine Wurzel eingeführt wurde: Onomatopoetika (gequietscht, pinged); Eigennamen (die Manns, the Childs); Entlehnungen (geoutet, succumbed; Mokassins, talismans)

- ein Wort unter Wortklassenwechsel gebildet wurde (gewachst, braked)

- ein komplexes Wort ohne einen "Kopf" erscheint und ihm daher semantische Information fehlt (gehaushaltet, grandstanded).

Natürlich werden unregelmäßige Wörter ebenfalls neugebildet, hauptsächlich durch Analogie, Zusammenfall oder als Restbestand früherer Regelmäßigkeit.

52 "Das Suffix -s übernahm die Vorherrschaft, weil es hörbar war, sich hinter Vokalen wie auch hinter Konsonanten sprechen ließ und, vor allem, in großer Zahl an Pluralnomen aus dem normannischen Französisch importiert wurde, das zufällig ebenso ein Pluralsuffix mit $-s$ besaß, und weil sie es an all jenen fremd klingenden Wörtern hörten, reanalysierten sie es als allmächtiges reguläres Suffix, das ganz allgemein an Nichtwurzeln treten konnte." (Pinker 2000: 272) 
cherwerb als übergroße Belastung ("learning burden"): Sprachwandel wird beschleunigt, der durch "Regularisierung" und Vereinfachung unsicher beherrschter Strukturen zum Abbau und mitunter zum Zusammenbruch des morphologischen Systems führen kann.

Die "Externalisierung" der Markierung externer Relationen (zum Beispiel Kasusrelationen) stellt einen Umstrukturierungsprozess dar, bei dem eine komplexe grammatische Information auf verschiedene Formen aufgeteilt wird, von denen jede nur eine geringe Informationsmenge trägt. Der in den Sprachinselvarietäten stattfindende sukzessive Abbau der Kasusmarkierung, ihre Ersetzung durch Artikel, dann durch Präpositionen, schließlich durch Wortstellungsregeln lassen sich interpretieren als fortgesetzte Transformation in Defaultregeln: Die Enkodierung "relationaler" Information durch Artikel (ohne morphologische Markierung oder mit vereinfachter), durch Präpositionen oder durch die Kombinationsregeln der Wortstellung sind ebenfalls "Operationen, die vorbehaltlos auf jedes Exemplar einer Kategorie anwendbar sind" (Pinker 2000: 269).

\section{$7 \quad$ Schluss}

Die untersuchten deutschen Sprachinselvarietäten zeigen heute massive Erscheinungen sprachlicher Konvergenz und sprachlichen Wandels. Um ihre sprachliche Entwicklung zu verstehen, haben wir zwischen intralingualer Konvergenz, interlingualer Konvergenz und typologischer "Konvergenz" (oder innersprachlichem Wandel) unterschieden sowie verwandte Phänomene wie "Pidginisierung" und "Regularisierung" (d.h. die Ausbreitung von regelmäßigen Bildungsmustern und deren Vereinfachung) betrachtet. Die Behandlung eines solchen komplexen Sachverhalts wie des Abbaus des morphologischen Systems in den Sprachinselvarietäten macht es notwendig, das Wechselverhältnis der beteiligten sprachlichen Prozesse zu bestimmen. Dafür scheint ein in doppeltem Sinne integrierter Ansatz sinnvoll:

Die Analyse der beteiligten sprachlichen Vorgänge, ihres jeweiligen Gewichts und ihres Zusammenspiels erfordert erstens die Einbeziehung von Methoden der Dialektologie, der Soziolinguistik, der Sprachkontakt- und der Sprachwandelforschung. (einschließlich einer Betrachtung der kognitiven Strukturen, die innerem Wandel unter äußerem Druck den Weg bahnen). Eine Theorie dieses Zusammenspiels könnte der Hauptgegenstand einer soziolinguistischen Sprachinselforschung sein.

Zweitens bietet sich eine vergleichende Sprachinselforschung an, um für die Analyse der beteiligten Prozesse Vergleichsfolien zu besitzen.

Die meisten deutschen Sprachinseln sind heute Sprachgemeinschaften in Auflösung. Der Abbau ihres grammatischen Systems ist diesbezüglich ihr gravierendstes Kennzeichen. Seit langem stehen sie in intensivem Sprachkontakt (und 
in einigen Sprachinseln ist auch der Varietätenkontakt von Bedeutung). Externer Einfluss ist folglich in nahezu allen deutschen Sprachinseln stark. Sprachliche Normen verblassen und geben Raum für Prozesse, die aus Pidginsprachen bekannt sind. Die Richtung des Sprachwandels und die "Anfälligkeit" bestimmter sprachlicher Strukturen für den Wandel sind hingegen in erster Linie durch systematische und typologische Strukturen determiniert, die nicht zuletzt kognitive Prozesse reflektieren, die auch dem "normalen" Wandel die Richtung geben.

Suzanne Romaine (1989: 380) bemerkt einigermaßen lakonisch, dass vielleicht "the kinds of changes which take place in language death are just ordinary changes speeded up". Nach der hier vorgenommenen Betrachtung lässt sich dies vermutlich - mit aller Vorsicht - bestätigen: Es ist mehr als eine Binsenweisheit: "in einem gewissen Sinne sind alle Entwicklungen 'intern"' (Coseriu 1975: 146).

\section{Literaturangaben}

Altenhofen, Cléo Vilson (1996): Hunsrückisch in Rio Grande do Sul. Ein Beitrag zur Beschreibung einer deutschbrasilianischen Dialektvarietät im Kontakt mit dem Portugiesischen. Stuttgart. (= Mainzer Studien zur Sprach- und Volksforschung. 21).

Andersen, Roger W. (1989): "The 'up' and 'down' staircase in secondary language development". In: Dorian, Nancy (ed.): Investigating obsolescence. Studies in language contraction and death. Cambridge etc.: 385-394.

Asfandiarova, Dania (1999): "'Mir verzehle doch Lutherisch und die Katholisch'. Dialektmischung im Vokalismus in der deutschen Sprachinsel Prišib/Aleksejevka (Baschkortostan, Russische Föderation)". In: Brandt, Gisela (ed.): Historische Soziolinguistik des Deutschen IV: Soziofunktionale Gruppe - Kommunikative Anforderungen - Sprachgebrauch. Intern. Fachtagung, Rostock, 13.-16.09.1998. Stuttgart: 241-262. (= Stuttgarter Arbeiten zur Germanistik. 372).

Askedal, John Ole (1996): "Überlegungen zum Deutschen als sprachtypologischem 'Mischtyp'". In: Lang, Ewald/Zifonun Gisela (eds.): Deutsch - typologisch. Berlin/ New York: 369-383. (= Institut für deutsche Sprache. Jahrbuch 1995).

Auer, Peter/Hinskens, Frans (1996): "The convergence and divergence of dialects in Europe. New and not so new developments in an old area". Sociolinguistica 10: $1-30$.

Berend, Nina/Jedig, Hugo (1991): Deutsche Mundarten in der Sowjetunion. Geschichte der Forschung und Bibliographie. Marburg.

Bohnenberger, Karl (1913): Die Mundart der deutschen Walliser im Heimattal und in den Aussenorten. Frauenfeld. (= Beiträge zur Schweizerdeutschen Mundart. 6).

Bourciez, Édouard (1967): Éléments de linguistique romane. Cinquième édition. Révisée par l'auteur et par les soins de Jean Bourciez. Paris.

Campbell, Lyle/Muntzel, Martha C. (1989): "The structural consequences of language death". In: Dorian, Nancy (ed.): Investigating obsolescence. Studies in language contraction and death. Cambridge etc.: 181-196. 
Cholewa, Jürgen (1993): "Störungen der lexikalisch-morphologischen Wortverarbeitung bei Aphasie: Ein Literaturüberblick". Neurolinguistik 1993, 7 (2): 105-126.

Clark, Thomas Lloyd (1972): Marietta, Ohio: The continuing erosion of a speech island. Alabama. (= Publication of the American Dialect Society. 57).

Comrie, Bernard (1996): "Sprache und Sprachen: Universalien und Typologie". In: Lang, Ewald/Zifonun, Gisela (eds.): Deutsch - typologisch.. Berlin, New York: 16-29. (= Institut für deutsche Sprache. Jahrbuch 1995).

Coseriu, Eugenio (1974): Synchronie, Diachronie und Geschichte. Das Problem des Sprachwandels. Übers. v. Helga Sohre. München.

Coseriu, Eugenio (1975): "Synchronie, Diachronie und Typologie". In: Cherubim, Dieter (ed.): Sprachwandel. Reader zur diachronischen Sprachwissenschaft. Berlin, New York: 135-149.

Deutscher Sprachatlas auf Grund des von Georg Wenker begründeten Sprachatlas des Deutschen Reichs und mit Einschluß von Luxemburg, der deutschen Sprachteile Österreichs, der Tschechoslowakei, der Schweiz, der Sprachinsel Gottschee, Liechtenstein in vereinfachter Form bearbeitet beim Deutschen Sprachatlas, begonnen von Ferdinand Wrede, fortgesetzt von Walther Mitzka und Bernhard Martin. 8. Lieferung. Marburg (Lahn) 1935.

Dinges, Georg (1925): "Zur Erforschung der wolgadeutschen Mundarten. (Ergebnisse und Aufgaben.)". Teuthonista 1/4: 299-313.

Dorian, Nancy (1989): "Introduction". In: Dorian, Nancy (ed.): Investigating obsolescence. Studies in language contraction and death. Cambridge etc.: 1-10.

Dressler, Wolfgang U./Wodak-Leodolter, Ruth (1977): "Language preservation and language death in Brittany". International Journal of the Sociology of Language 12: $33-44$.

Dulson, Andreas (1941): "Problema skreschtschenija dialektow po materialam jasyka nemzew Powolshja". Iswestija Akademii nauk Sojusa SSR, Otdelenie literatury $i$ jasyka 3: 82-96.

Eisenberg, Peter (1994): "German". In: König, Ekkehard/van der Auwera, Johan (eds.): The Germanic Languages. London, New York: 349-387.

Esser, Hartmut (1996a): "Die Definition der Situation". Kölner Zeitschrift für Soziologie und Sozialpsychologie 48: 1-34.

Esser, Hartmut (1996b): "Ethnische Konflikte als Auseinandersetzung um den Wert von kulturellem Kapital". In: Heitmeyer, Wilhelm/Dollase, Rainer (eds.): Die bedrängte Toleranz. Ethnisch-kulturelle Konflikte, religiöse Differenzen und die Gefahren politisierter Gewalt. Frankfurt/Main: 64-99.

Giles, Howard/Coupland, Nikolas (1991): Language: Contexts and Consequences. Milton Keynes, Pacific Grove, CA.

Grinjowa, Nelly M. (1990): "Interferenzerscheinungen im grammatischen System einer niederdeutschen Mundart in der Sowjetunion infolge intensiver Sprachkontakte". (ms., unveröff.).

Gumperz, John J. (1968): "The speech community". In: Grignioli, P. P. (ed.): Language and Social Context. Harmondsworth: 219-231.

Haberland, Hartmut (1994): "Danish". In: König, Ekkehard/van der Auwera, Johan (eds.): The Germanic Languages. London, New York: 313-348. 
Harwood, Jake/Giles, Howard/Bourhis, Richard Y. (1994): "The genesis of vitality theory: historical patterns and discoursal dimensions". International Journal of the Sociology of Language 108: 167-206.

Haugen, Einar (1989): "The rise and fall of an immigrant language: Norwegian in America". In: Dorian, Nancy (ed.): Investigating obsolescence. Studies in language contraction and death. Cambridge etc.: 61-73.

Hinskens, Frans/Kallen, Jeffrey L./Taeldeman, Johan (2000): "Merging and drifting apart. Convergence and divergence of dialects across political borders". International Journal of the Sociology of Language 145: 1-28.

Hornung, Maria (1994): "Die sogenannten zimbrischen Mundarten der Sieben und Dreizehn Gemeinden in Oberitalien". In: Hornung, Maria (ed.) (1994): Die deutschen Sprachinseln in den Südalpen: Mundarten und Volkstum. Hildesheim, Zürich, New York: 19-43. (= Studien zur Dialektologie. 3).

Huffines, Marion L. (1989): "Case usage among the Pennsylvania German sectarians and nonsectarians". In: Dorian, Nancy (ed.): Investigating obsolescence. Studies in language contraction and death. Cambridge etc.: 211-226.

Huffines, Marion L. (1994): "Directionality of Language Influence: The Case of Pennsylvania German and English". In: Berend, Nina/Mattheier, Klaus J. (eds.): Sprachinselforschung. Eine Gedenkschrift für Hugo Jedig. Frankfurt/Main: 47-58.

Hunnius, Klaus (1990): "Französisch: Flexionslehre". In: Holtus, Günter/Metzeltin, Michael/Schmitt, Christian (eds.): Lexikon der Romanistischen Linguistik (LRL). Bd./Volume V,1: 59-71.

Hutterer, Claus J. (1982): "Sprachinselforschung als Prüfstand für dialektologische Arbeitsprinzipien". In: Besch, Werner/Knoop, Ulrich/Putschke, Wolfgang/Wiegand, Herbert Ernst (eds.): Dialektologie. Ein Handbuch zur deutschen und allgemeinen Dialektforschung. 2. Halbbd. Berlin, New York: 178-189.

Hutterer, Claus J. (1987): Die germanischen Sprachen. Ihre Geschichte in Grundzügen. 2. Aufl. (1. Aufl. 1975). Budapest.

Jakobson, Roman (1936): "Beitrag zur allgemeinen Kasuslehre. Gesamtbedeutungen der russischen Kasus". Travaux du Cercle Linguistique de Prague 6: Études Dédiées au Quatrième Congrès de Linguistes, Prague 1936. Praha: 240-288.

Jakobson, Roman (1969): Kindersprache, Aphasie und allgemeine Lautgesetze. Frankfurt/Main.

Jedig, Hugo H. (1966): Laut- und Formenbestand der niederdeutschen Mundart des Altai-Gebietes. Berlin. (= Sitzungsberichte der Sächsischen Akademie der Wissenschaften zu Leipzig. Philologisch-historische Klasse. 112/5).

Kaufmann, Göz (1997): Varietätendynamik in Sprachkontaktsituationen. Attitüden und Sprachverhalten rußlanddeutscher Mennoniten in Mexiko und den USA. Frankfurt am Main etc. (= VarioLingua. 3)

Keel, William D. (1994): "Reduction and Loss of Case Marking in the Noun Phrase in German-American Speech Islands: Internal Development or External Interference?". In: Berend, Nina/Mattheier, Klaus J. (eds.): Sprachinselforschung. Eine Gedenkschrift für Hugo Jedig. Frankfurt/Main: 93-104.

König, Ekkehard (1996): "Kontrastive Grammatik und Typologie". In: Lang, Ewald/Zifonun Gisela (eds.): Deutsch - typologisch. Berlin, New York: 31-54. (= Institut für deutsche Sprache. Jahrbuch 1995). 
König, Ekkehard/van der Auwera, Johan (eds.) (1994): The Germanic Languages. London, New York.

Kuhn, Walter (1934): Deutsche Sprachinsel-Forschung. Geschichte, Aufgaben, Verfahren. Plauen i. Vogtl. (=Ostdeutsche Forschungen. 2).

Lang, Ewald (1996): "Das Deutsche im typologischen Spektrum. Einführung in den Band". In: Lang, Ewald/Zifonun Gisela (eds.): Deutsch - typologisch. Berlin, New York: 7-15. (=Institut für deutsche Sprache. Jahrbuch 1995).

Langacker, Ronald (1999): Grammar and Conceptualization. Berlin, New York. (= Cognitive Linguistics Research. 14)

Lapinski, Dariusz/Rosenberg, Peter (2001): "Sprachnationalismus und die 'Logik' ethnosprachlicher Konflikte". Frankfurt (Oder). Vortrag, gehalten auf der Jahrestagung 2002 der Gesellschaft für Angewandte Linguistik (GAL), Passau, 27.-29.09.2001. (Ms., unveröff.).

Lausberg, Heinrich (1962): Romanische Sprachwissenschaft. Vol. III: Formenlehre. Zweiter Teil. Berlin.

Le Page, Robert B./Tabouret-Keller, Andrée (1985): Acts of Identity. Creole-based approaches to language and ethnicity. Cambridge usw.

Louden, Mark L. (1994): "Syntactic Change in Multilingual Speech Islands". In: Berend, Nina/Mattheier, Klaus J. (eds.): Sprachinselforschung. Eine Gedenkschrift für Hugo Jedig. Frankfurt/Main: 73-91.

Mattheier, Klaus J. (1996): "Methoden der Sprachinselforschung". In: Goebl, Hans/ Nelde, Peter H./Starý, Zdenek/Wölck, Wolfgang (eds.) (1996/97): Kontaktlinguistik/ Contact linguistics/Linguistique de contact. Ein internationales Handbuch zeitgenössischer Forschung. An international handbook of contemporary research. Manuel international des recherches contemporaines. 1. Halbbd. Berlin, New York: 812-819.

Mayerthaler, Willi/Fliedl, Günther/Winkler, Christian (1998): Lexikon der Natürlichkeitsheoretischen Syntax und Morphosyntax. Tübingen.

Milroy, Lesley (1980): Language and Social Networks. Oxford.

Mufwene, Salikoko S. (1998): "What research on Creole Genesis Can Contribute to Historical Linguistics". Historical Linguistics. Selected Papers from the $13^{\text {th }}$ International Conference on Historical Linguistics, Düsseldorf, 10-17 August 1997, ed. by Monika S. Schmid, Jennifer R. Austin, Dieter Stein. Amsterdam, Philadelphia: 315-338.

Niedzielski, Nancy/Giles, Howard (1996): "Linguistic accomodation". In: Goebl, Hans/ Nelde, Peter H./ Starý, Zdenek/Wölck, Wolfgang (eds.) (1996/97): Kontaktlinguistik/Contact linguistics/Linguistique de contact. Ein internationales Handbuch zeitgenössischer Forschung. An international handbook of contemporary research. Manuel international des recherches contemporaines. 1. Halbbd. Berlin, New York: de Gruyter: 332-342.

Nöth, Dorothea (1994): Spuren der verlernten Muttersprache? Beobachtungen zum Artikelgebrauch beim Sprachwiedererwerb von Rußlanddeutschen. (ms., unveröff.). Berlin.

Nyman, Lennart (1997): "Einige Beobachtungen zu Varietäten und Varietätenausgleich im Niederdeutsch der Orenburger Rußland-Mennoniten". In: Brandt, Gisela (ed.): Historische Soziolinguistik des Deutschen III. Sprachgebrauch und sprachliche 
Leistung in sozialen Schichten und soziofunktionalen Gruppen. Internationale Fachtagung Rostock/Kühlungsborn 15.-18.9.1996. Stuttgart: 261-276.

O'Neil, Wayne (1978): "The Evolution of the Germanic Inflectional Systems: A Study in the Causes of Language Change". Orbis 27: 248-286.

Pinker, Steven (2000): Wörter und Regeln. Die Natur der Sprache. Aus dem Englischen übersetzt von Martina Wiese. Heidelberg, Berlin.

Romaine, Suzanne (1989): "Pidgins, Creoles, immigrant, and dying languages". In: Dorian, Nancy (ed.): Investigating obsolescence. Studies in language contraction and death. Cambridge etc.: 369-383.

Rosenberg, Peter (1986): Der Berliner Dialekt - und seine Folgen für die Schüler. Tübingen.

Rosenberg, Peter (1994): "Sprachgebrauchsstrukturen und Heterogenität der Kommunikationsgemeinschaft bei den Deutschen in der GUS - eine empirische Studie". In: König, Peter-Paul/Wiegers, Helmut (eds.): Satz - Text-Diskurs. Akten des 27. Linguistischen Kolloquiums, Münster 1992. Bd. 2. Tübingen: 287-298.

Rosenberg, Peter (1998): "Deutsche Minderheiten in Lateinamerika". In: Harden, Theo/Hentschel, Elke (eds.): Particulae particularum. Festschrift zum 60. Geburtstag von Harald Weydt. Tübingen 1998: 261-291.

Rosenberg, Peter (2003): "Dialect convergence in German speech islands". In: Auer, Peter/Hinskens, Frans/Kerswill, Paul E. (eds.): Dialect convergence and divergence in Europe. (in Vorber.)

Rosenberg, Peter (ersch. demn.): "Die wolgadeutsche Dialektologie und die Kampagne gegen den 'Nationalismus'". Deutsche in der Sowjetunion 1929-1941. Beiträge der wissenschaftlichen Fachtagung des Göttinger Arbeitskreises e.V. in Zusammenarbeit mit der Wissenschaftlichen Kommission für die Deutschen in Rußland und in der GUS, Göttingen-Bovenden, 30.10.-1.11.1997.

Salmons, Joseph (1994): "Naturalness and Morphological Change in Texas German". In: Berend, Nina/Mattheier, Klaus J. (eds.): Sprachinselforschung. Eine Gedenkschrift für Hugo Jedig. Frankfurt/Main: 59-72.

Schmeller, Johann Andreas (1855): Sogenanntes Cimbrisches Wörterbuch, das ist deutsches Idioticon der VII und XIII communi in den venetianischen Alpen. Mit Einleitung und Zusammenfassung im Auftrag der Kaiserlichen Akademie der Wissenschaften. Hrsgg. von J. Bergmann. Wien.

Schirmunski, Viktor M. (1930): "Sprachgeschichte und Siedelungsmundarten". Germanisch-Romanische Monatsschrift 18, H. 3/4: 113-122; H. 5/6: 171-188.

Schirmunski, Viktor M. (1962): Deutsche Mundartkunde. Vergleichende Laut- und Formenlehre der deutschen Mundarten. Berlin.

Smits, Caroline (1996): Disintegration of Inflection. The Case of Iowa Dutch. Amsterdam.

Smits, Caroline (1998): "Two Models for the Study of Language Contact: A PsychoLinguistic Perspective Versus a Socio-Cultural Perspective". In: Schmid, Monika S./Jennifer R. Austin/Dieter Stein (eds.): Historical Linguistics. Selected Papers from the $13^{\text {th }}$ International Conference on Historical Linguistics, Düsseldorf, 10-17 August 1997. Amsterdam, Philadelphia: 377-390.

Trudgill, Peter (1978): "Creolization in Reverse". Transactions of the Philological Society 1976/77: 32-50. 
van Coetsem, F. (1995): "Outlining a model of the transmission phenomenon in language contact". Leuvense Bijdragen 84, 1: 63-85.

van der Plank, Pieter H. (1978): "The Assimilation and Non-Assimilation of European Linguistic Minorities. A Sociological Retrospection". In: Fishman, Joshua A. (ed.): Advances in the Study of Societal Multilingualism. The Hague, Paris, New York: 423-456. (= Contributions to the Sociology of Language. 9).

van Kemenade, Ans. (1994): "Old and Middle English". In: König, Ekkehard/van der Auwera, Johan (eds.): The Germanic Languages. London, New York: 110-141.

van Ness, Silke (1994): "Pennsylvania German". In: König, Ekkehard/van der Auwera, Johan (eds.): The Germanic Languages. London, New York: 420-438.

Weinreich, Uriel/Labov, William/Herzog, Marvin I. (1968): "Empirical Foundations for a Theory of Language Change". In: Lehmann, W. P./Malkiel, Yakov (eds.): Directions for Historical Linguistics: A Symposium. Austin, London: 95-188.

Weinrich, Harald (1985): Textgrammatik der französischen Sprache. 1. Aufl. 1982, Nachdruck. Stuttgart.

Wiesinger, Peter (1983): "Deutsche Dialektgebiete außerhalb des deutschen Sprachgebiets: Mittel-, Südost- und Osteuropa". In: Besch, Werner/Knoop, Ulrich/ Putschke, Wolfgang/Wiegand, Herbert Ernst (eds.): Dialektologie. Ein Handbuch zur deutschen und allgemeinen Dialektforschung. 2. Halbbd. Berlin, New York: 900-929.

Wolgadeutscher Sprachatlas (WDSA), aufgrund der von Georg Dinges 1925-1929 gesammelten Materialien. Bearb. und hrsg. von Nina Berend. Unter Mitarb. von Rudolf Post. Tübingen, Basel 1996.

Wurzel, Wolfgang U. (1996): "Morphologischer Strukturwandel: Typologische Entwicklungen im Deutschen". In: Lang, Ewald/Zifonun Gisela (eds.): Deutsch - typologisch. Berlin, New York: 492-524. (= Institut für deutsche Sprache. Jahrbuch 1995). 
Kasussystem der Personalpronomina im Pennsylvania German

(cf. van Ness 1994: 430; Huffines 1989: 216)

\begin{tabular}{|c|c|c|c|}
\hline Person & Nominativ & Akkusativ & Dativ \\
\hline \multirow[t]{2}{*}{ 1. Sing. } & iç & miç & $\begin{array}{c}\text { mir } \\
\text { mer (unbetont) }\end{array}$ \\
\hline & 'ich' & 'mich' & 'mir' \\
\hline \multirow[t]{2}{*}{ 2. Sing. } & $\mathrm{du}$ & diç & $\begin{array}{c}\text { dir } \\
\text { der (unbetont) }\end{array}$ \\
\hline & 'du & 'dich & 'dir' \\
\hline \multirow[t]{2}{*}{ 3. Sing. Mask. } & ar & $\begin{array}{c}\text { in } \\
\text { en (unbetont) }\end{array}$ & $\begin{array}{c}\text { im } \\
\text { em (unbetont) }\end{array}$ \\
\hline & 'er' & 'ihn & 'ihm' \\
\hline \multirow[t]{2}{*}{ 3. Sing. Fem. } & si & si & $\begin{array}{c}\text { ire/ir } \\
\text { re (unbetont) }\end{array}$ \\
\hline & 'sie & 'sie & 'ihr' \\
\hline \multirow[t]{2}{*}{ 3. Sing. Neutr. } & es & es & $\begin{array}{c}\mathrm{im} \\
\text { em (unbetont) }\end{array}$ \\
\hline & 'es' & 'es' & 'ihm' \\
\hline \multirow[t]{2}{*}{ 1. Plur. } & $\begin{array}{c}\text { mir } \\
\text { mer (unbetont) }\end{array}$ & uns & uns \\
\hline & 'wir' & 'uns & 'uns' \\
\hline \multirow[t]{2}{*}{ 2. Plur. } & $\begin{array}{c}\text { dir/ir } \\
\text { der/er/ner/nir (un- } \\
\text { betont) }\end{array}$ & aiç & aiç \\
\hline & 'ihr' & 'euch & 'euch \\
\hline \multirow[t]{2}{*}{ 3. Plur. } & si & si & $\begin{array}{c}\text { ine } \\
\text { ne (unbetont) }\end{array}$ \\
\hline & 'sie & 'sie & 'ihnen \\
\hline
\end{tabular}


Kasussystem der Personalpronomina im Altenglischen/Neuenglischen (nach van Kemenade 1994: 121)

\begin{tabular}{|c|c|c|c|c|}
\hline Person & Nominativ & Akkusativ & Dativ & Genitiv \\
\hline \multirow[t]{2}{*}{ 1. Sing. } & ic & me: & me: & mi:n \\
\hline & 'I' & 'me' & 'me' & 'my/of me' \\
\hline \multirow[t]{2}{*}{ 2. Sing. } & u & e: & e: & i:n \\
\hline & 'you' & 'you' & 'you' & 'your/of you' \\
\hline \multirow[t]{2}{*}{ 3. Sing. Mask. } & he: & hine & him & his \\
\hline & 'he' & 'him' & 'him' & 'his/of him' \\
\hline \multirow[t]{2}{*}{ 3. Sing. Fem. } & he:o & hi: & hire & hire \\
\hline & 'she' & 'her' & 'her' & 'her/of her' \\
\hline \multirow[t]{2}{*}{ 3. Sing. Neutr. } & hit & hit & him & his \\
\hline & 'it' & 'it' & 'it' & 'its/of it' \\
\hline \multirow[t]{2}{*}{ 1. Plur. } & we: & $\mathrm{u}: \mathrm{s}$ & $\mathrm{u}: \mathrm{s}$ & u:re \\
\hline & 'we' & 'us' & 'us' & 'our/of us' \\
\hline \multirow[t]{2}{*}{ 2. Plur. } & ge: & e:ow & e:ow & e:ower \\
\hline & 'you' & 'you' & 'you' & 'your/of you' \\
\hline \multirow[t]{2}{*}{ 3. Plur. } & hi: & hi: & him & hira \\
\hline & 'they' & 'them' & 'them' & 'their/of them' \\
\hline
\end{tabular}


Kasussystem der Personalpronomina im Dänischen (nach Haberland 1994: 328)

\begin{tabular}{|c|c|c|c|}
\hline Person & $\begin{array}{c}\text { Non- } \\
\text { Obliquenkasus }\end{array}$ & Obliquenkasus & Possessiv \\
\hline \multirow[t]{2}{*}{ 1. Sing. } & jeg & mig & $\min$ \\
\hline & 'ich' & 'mir/mich' & 'mein-' \\
\hline \multirow[t]{2}{*}{ 2. Sing. } & $\mathrm{du}$ & dig & $\operatorname{din}$ \\
\hline & 'du' & 'dir/dich' & 'dein-' \\
\hline \multirow[t]{2}{*}{ 3. Sing. Mask. } & han & $\begin{array}{l}\text { ham } \\
\text { altskand. Dativ: } \\
\text { honum }\end{array}$ & hans \\
\hline & 'er' & 'ihm/ihn' & 'sein-' \\
\hline \multirow[t]{2}{*}{ 3. Sing. Fem. } & hun & $\begin{array}{l}\text { hende [hinne] } \\
\text { altskand. Dativ: } \\
\text { henni }\end{array}$ & hendes \\
\hline & 'sie' & 'ihr/sie' & 'ihr-' \\
\hline \multirow{2}{*}{$\begin{array}{l}\text { 3. Sing. Neutr. } \\
\text { [- menschl.] }\end{array}$} & det & det & dets \\
\hline & 'es' & 'ihm/es' & 'sein-' \\
\hline \multirow{2}{*}{$\begin{array}{c}\text { 3. Sing. Non-Neutr. } \\
\text { [- menschl. }]\end{array}$} & den & den & dens \\
\hline & ('er, sie, es') & $\begin{array}{c}\text { ('ihm/ihn, ihr/sie, } \\
\text { ihm/es') }\end{array}$ & $\begin{array}{l}\text { ('sein-, ihr-, } \\
\text { sein-') }\end{array}$ \\
\hline \multirow[t]{2}{*}{ 1. Plur. } & vi & os & vores/vor \\
\hline & 'wir' & 'uns' & 'unser-' \\
\hline \multirow[t]{2}{*}{ 2. Plur. } & I & jer & jeres \\
\hline & 'ihr' & 'euch' & 'euer-' \\
\hline \multirow[t]{2}{*}{ 3. Plur. } & de $[\mathrm{di}]$ & $\begin{array}{c}\text { dem } \\
\text { altskand. Dativ: } \\
\text { eim } \\
\text { (Demonstra- } \\
\text { tivpron., Distal) }\end{array}$ & deres \\
\hline & 'sie' & 'ihnen/sie' & 'ihr-' \\
\hline
\end{tabular}


Kasussystem der "pronoms personnels conjoints" im Französischen (nach Weinrich 1985: 82)

\begin{tabular}{|c|c|c|c|}
\hline & Subjekt & Objekt & Partner \\
\hline \multirow{2}{*}{$\begin{array}{c}\text { Sprecher } \\
\text { (Sing.) } \\
\end{array}$} & je & $\mathrm{me} / \mathrm{m}^{\prime}$ & $\mathrm{me} / \mathrm{m}^{\prime}$ \\
\hline & 'ich' & 'mich' & 'mir' \\
\hline \multirow{2}{*}{$\begin{array}{c}\text { Adressat } \\
\text { (Sing.) }\end{array}$} & tu & te $/ t^{\prime}$ & te $/ t^{\prime}$ \\
\hline & 'du' & 'dich' & 'dir' \\
\hline \multirow{2}{*}{$\begin{array}{c}\text { Referenz (mask.) } \\
\text { (Sing.) }\end{array}$} & il & le/1' & lui \\
\hline & 'er' & 'ihn' & 'ihm' \\
\hline \multirow{2}{*}{$\begin{array}{c}\text { Referenz (fem.) } \\
\text { (Sing.) }\end{array}$} & elle & $\mathrm{la} / \mathrm{l}^{\prime}$ & lui \\
\hline & 'sie' & 'sie' & 'ihr' \\
\hline \multirow{2}{*}{$\begin{array}{c}\text { Sprecher } \\
\text { (Plur.) } \\
\end{array}$} & nous (on) & nous & nous \\
\hline & 'wir' & 'uns' & 'uns' \\
\hline \multirow{2}{*}{$\begin{array}{c}\text { Adressat } \\
\text { (Plur.) }\end{array}$} & vous & vous & vous \\
\hline & 'ihr/Sie' & 'euch/Sie' & 'euch/Ihnen' \\
\hline \multirow{2}{*}{$\begin{array}{c}\text { Referenz (mask.) } \\
\text { (Plur.) }\end{array}$} & ils & les & leur \\
\hline & 'sie' & 'sie' & 'ihnen' \\
\hline \multirow{2}{*}{$\begin{array}{c}\text { Referenz (fem.) } \\
\text { (Plur.) }\end{array}$} & elles & les & leur \\
\hline & 'sie' & 'sie' & 'ihnen' \\
\hline
\end{tabular}


Lexikalische Repräsentation von Wörtern: "Dekomposition" versus "Full Listing"

[cf. Cholewa (1993)]

\begin{tabular}{|c|c|}
\hline DEKOMPOSITION & FULL LISTING \\
\hline polymorphematische Wörter: & polymorphematische Wörter: \\
\hline reguläre Wortbildung & irreguläre Wortbildung \\
\hline $\begin{array}{c}\text { produktive morphologische Paradig- } \\
\text { men }\end{array}$ & $\begin{array}{c}\text { weniger produktive morph. Paradig- } \\
\text { men }\end{array}$ \\
\hline $\begin{array}{c}\text { semantisch transparente Wortstruktur } \\
\text { semantisch weniger transparente } \\
\text { Wortstruktur }\end{array}$ \\
\hline hochfrequente Morpheme & niedrigfrequente Morpheme \\
\hline
\end{tabular}


particulae collectae 